\title{
Aldehyde Dehydrogenase 2 Activator Augments the Beneficial Effects of Empagliflozin in Diabetes Aassociated HFpEF
}

\section{Guodong Pan}

Henry Ford Health System

Bipradas Roy

Wayne State University

Shailendra Giri

Henry Ford Health System

David E Lanfear

Henry Ford Health System

Rajarajan A. Thandavarayan

Houston Methodist Research Institute

Ashrith Guha

Houston Methodist Research Institute

Pablo Ortiz

Henry Ford Health System

Suresh Selvaraj Palaniyandi ( $\sim$ spalani2@hfhs.org )

Henry Ford Health System

\section{Research Article}

Keywords: Diabetic HFpEF, 4-hydroxy-2-nonenal, Aldehyde dehydrogenase 2, Empagliflozin, Alda-1, Cardiac function and ALDH2 * 2 mutant mice

Posted Date: March 8th, 2022

DOI: https://doi.org/10.21203/rs.3.rs-1362749/v2

License: (c) (i) This work is licensed under a Creative Commons Attribution 4.0 International License.

Read Full License 


\section{Abstract}

Objectives: To ameliorate diabetes mellitus associated heart failure with preserved ejection fraction (HFpEF), we plan to lower diabetes-mediated oxidative stress-induced 4-hydroxy-2-nonenal (4HNE) accumulation by pharmacological agents that either decrease 4HNE generation or increase its detoxification.

Background: 4HNE, a cellular reactive carbonyl species (RCS), was significantly increased in diabetic hearts due to a diabetes-induced decrease in 4HNE detoxification by aldehyde dehydrogenase (ALDH)2, a cardiac mitochondrial enzyme that metabolizes $4 \mathrm{HNE}$. Therefore, hyperglycemia-induced $4 \mathrm{HNE}$ is critical for diabetes-mediated cardiotoxicity and we hypothesize that lowering 4HNE ameliorates diabetes associated HFpEF.

Methods: We fed high-fat diet to ALDH2*2 mice which have intrinsically low ALDH2 activity to induce type-2 diabetes. After 4 months of diabetes, the mice exhibited features of HFpEF and along with increased $4 \mathrm{HNE}$ adducts and we treated them with vehicle, empagliflozin (EMP) $(3 \mathrm{mg} / \mathrm{kg} / \mathrm{d})$ to reduce $4 \mathrm{HNE}$ and Alda- 1 (10mg/kg/d), an ALDH2 activator to enhance ALDH2 activity as well as a combination of EMP + Alda-1 $(E+A)$, via subcutaneous osmotic pumps. After 2 months of treatments, cardiac function was assessed by conscious echocardiography before and after exercise stress.

Results: EMP + Alda-1 improved exercise tolerance, diastolic and systolic function, 4HNE detoxification and cardiac liver kinase B1 (LKB1)-AMP-activated protein kinase (AMPK) pathway in ALDH2*2 mice with diabetes associated HFpEF. This combination was even more effective than EMP alone.

Conclusions: Our data indicate that ALDH2 activation along with the treatment of hypoglycemic agents may be a salient strategy to alleviate diabetes associated HFpEF.

\section{Highlights}

- Combined treatment of EMP, a sodium-glucose cotransporter (SGLT) 2 inhibitor and Alda-1, an aldehyde dehydrogenase (ALDH)2 activator improved exercise tolerance, diastolic function as well as cardiac hypertrophy and fibrosis in ALDH2*2 mutant diabetic mice exhibiting HFpEF features.

- The ameliorative effects of EMP + Alda-1 combination is greater than EMP alone.

- EMP + Alda-1 combination significantly decreased 4-hydroxy-2-nonenanl (4HNE) protein adducts in the hearts of ALDH2*2 mutant diabetic mice exhibiting HFpEF features.

- Decreasing 4HNE protein adduct levels by two different pharmacological approaches improved phosphorylation of cardiac liver kinase (LK) B1 and its substrate AMP-activated protein kinase (AMPK) with a decrease in LKB1-4HNE adducts.

\section{Introduction}


Multiple factors contribute to heart failure with preserved ejection fraction (HFpEF), a condition that remains poorly understood and difficult to study or treat. The protean nature of HFpEF makes it unsurprising that there is not a single animal model that can mimic all the characteristics of HFpEF [1]. However, different preclinical models can recapitulate different features of HFpEF based on etiology. This can be cleverly segregated into individual models of HFpEF based on their etiopathophysiology, such as models of aging mediated HFpEF, hypertension mediated HFpEF, atrial fibrillation mediated HFpEF, pulmonary hypertension mediated HFpEF, obesity mediated HFpEF and diabetes mediated HFpEF [1]. In this study, our focus will be on HFpEF associated with type-2 diabetes mellitus (type $2 \mathrm{DM}$ ). There is no crystal-clear understanding of the pathophysiology of HFpEF associated with type $2 \mathrm{DM}$. However, the emerging consensus in the research field is that DM mediated systemic oxidative stress, inflammation and metabolic disturbances set the stage for end-organ damages including HFpEF.

Among the various factors in diabetes, hyperglycemia mediated oxidative stress has been regarded as critical in the diabetes-mediated end-organ damage via multiple pathways [2,3]. Oxidative stress is a complex and continuous process. Reactive oxygen species (ROS) generation is the first event of oxidative stress; however, ROS have short half-life. But to further continue the oxidative stress process, ROS produce reactive carbonyl species (RCS) such as 4-hydroxy-2-nonenal (4HNE) by attacking the biological membranes a phenomenon known as lipid peroxidation. Thus, RCS are usually regarded as secondary messengers of oxidative stress, which form adducts with cellular macromolecules that lead to organ damage. RCS exert either biological, physiological, cytotoxic and/or genotoxic properties based on their concentration at the tissue or the systemic level. For instance, when diabetic complications ensue in long standing diabetes, apart from lipid peroxidation, nonenzymatic (glycation and amino acid oxidation) and enzymatic (polyol pathway and glucose oxidation) reactions also generate RCS [3].

Increased accumulation of RCS including reactive aldehydes and ketones in both type-1 and type-2 diabetes mellitus has been well documented [4-6]. For instance, reactive aldehydes such as acrolein, malondialdehyde, glyoxal and 4-hydroxy-2-nonenal (4HNE) are increased in diabetic tissue [7]. 4HNE is known to form adducts with proteins, DNA and extracellular matrix and we have shown increased 4HNE adducts in both type-1 and type-2 diabetic hearts compared to controls [8-10], which contribute to cardiac damage and dysfunction [4]. Thus, interventions to reduce the accumulation of $4 \mathrm{HNE}$ either by decreasing its generation or enhancing its detoxification have potential to protect the myocardium from diabetes-related damage.

In myocardial cells, $4 \mathrm{HNE}$ is detoxified by glutathione, aldehyde dehydrogenases (ALDHs) and aldose reductase [11]. We showed a significant increase in 4HNE adducts in cultured cardiac cells [12-15] and cardiac tissue $[8-10,16]$, which is augmented by reduced ALDH2 activity by employing either diabetic insult or pharmacological inhibition or genetic mutation. A single point E487K mutation in ALDH2 (prevalent in East Asians) known as ALDH2*2 reduces its activity to 3\% in homozygotes (ALDH2*2/2) and $~ 30-50 \%$ in heterozygotes (ALDH2*2/1). We employed ALDH2*2 knock-in heterozygous mutant mice which mimic East Asian with ALDH2*2 mutation having intrinsically low ALDH2 activity. 
Since ALDH2*2 carriers are prevalent in East Asia, which contains a growing diabetic population, our study will be helpful in managing diabetic cardiac complications including HFpEF. In fact, a recent clinical study in East Asians with ALDH2*2 mutation demonstrated an association between ALDH2*2 and HFpEF in patients having diabetes and other comorbidities [17]. Even before this study, we were the first to demonstrate that ALDH2*2 mutant mice with type-2 DM exhibited exacerbation of HFpEF features relative to wild-type mice with type-2 DM [10]. Thus, we employed the same high-fat diet (HFD)-fed ALDH2*2 mutant diabetic mice in this study as well.

In this study, we plan to only use pharmacological approaches as the majority of current therapeutic management is still implemented through drugs. In our earlier study, we decreased cardiac $4 \mathrm{HNE}$ adduct levels in ALDH2*2 diabetic mice by decreasing hyperglycemia via empagliflozin (EMP), a sodium-glucose cotransporter (SGLT) 2 inhibitor and thereby improved cardiac function via altering metabolic signaling [18]. EMP like SGLT2 inhibitors are shown to ameliorate HF in patients with diabetes and other metabolic conditions and several mechanisms have been proposed for this beneficial effect $[19,20]$. As we have found recently that EMP was effective in lowering $4 \mathrm{HNE}$ levels, we plan to augment the attenuation of cardiac $4 \mathrm{HNE}$ adduct levels in the current study. Therefore, in addition to decreasing 4HNE generation via EMP, we also want to increase 4HNE detoxification via ALDH2 activation by Alda-1, a small molecule ALDH2 activator. Alda- 1 was first shown to increase both wild type (WT) ALDH2 and ALDH2*2 activity and decrease $4 \mathrm{HNE}$ adductions in vitro and in the myocardium of rats subjected to ischemia-reperfusion [21]. Also, Alda-1 has been used for the treatment of diabetes-induced cardiac damage as well [22, 23]. We recently show that Alda-1 protected the exogenous $4 \mathrm{HNE}$-induced myocardial toxicity and it also prevented myocardial ischemia-reperfusion induced damage in both WT and ALDH2*2 mice [24].

Therefore, in this study, we will test the hypothesis that combining both lowering cardiac 4HNE adducts by reducing hyperglycemia-mediated oxidative stress and increasing 4HNE detoxification by ALDH2 activation should ameliorate DM associated HFpEF in ALDH2*2 mice. We want to focus on cardiac function and exercise tolerance along with cardiomyocyte hypertrophy and fibrosis as endpoints since HFpEF patients exhibit poor quality of life such as exercise intolerance and cardiac dysfunction with underlying cardiac pathological remodeling.

\section{Materials And Methods}

High-fat diet (HFD)-fed type-2 diabetes mellitus in ALDH2*2 mutant mice.

ALDH2*2 knock-in mutant mice (with C57BL/6J background) either male or female around 3-4 months of age were fed an HFD (60\% of calories from fat, D12492, Research Diets) as we published earlier [10]. Normal chow fed ALDH2*2 mice were served as controls. In the HFD-fed group, the mice with sustained elevated fasting blood glucose levels above $250 \mathrm{mg} / \mathrm{dl}$ were deemed as diabetic mice and selected for further treatments. ALDH2*2 mice were inbred in-house and genotyped by Transnetyx Inc. The animal protocol was approved by the Henry Ford Health System and Wayne State University Institutional Animal 
Care and Use Committee. We confirm that all experiments were performed in accordance with IACUC guidelines and regulations. All the animal experiments followed the ethical guidelines of ARRIVE.

\section{Intraperitoneal glucose tolerance test (IPGTT)}

The IPGTT was performed in mice from control and diabetic groups as explained elsewhere [8]. After fasting for $6 \mathrm{~h}$, the mice were injected with $2 \mathrm{~g} / \mathrm{kg}$ D-glucose. Then, blood glucose levels were measured at 0, 30, 60, 90 and 120 min after D-glucose injection, using a glucometer.

\section{Pharmacological Treatment Protocols}

At the end of 6 months, we divided the diabetic ALDH2 22 mice randomly into 4 groups with $\mathrm{N}=8$ animals for each group: DMSO as vehicle (Veh); an SGLT2 inhibitor, EMP (3mg/ $\mathrm{kg} / \mathrm{d})$ to reduce hyperglycemia; an ALDH2 activator, Alda- 1 (10mg/ $\mathrm{kg} / \mathrm{d}$ ) to enhance ALDH2 activity (Alda-1); as well as a combination of EMP + Alda-1. These agents were administered via using Alzet Mini-osmotic pumps (Model \# 2004; 0.25 $\mu \mathrm{l}$ per hour, 28 days) by implanting subcutaneously (we changed the pumps after 28 days for one more time to administer for 56 days (i.e., 8 weeks). We selected the dose of EMP from previously published studies from us [18] and others [25-27]. Similarly, Alda-1 dose was selected from previous studies [2830]. After 8 weeks of treatments, cardiac function was assessed by conscious echocardiography postexercise stress.

\section{Cardiac function assessment by echocardiography in conscious mice}

To avoid the effects of anesthesia, we assessed left ventricular dimension and function in conscious mice using an echocardiograph equipped with a 15-MHz linear transducer (Acuson c256) as we described previously $[8,10,31]$.

Acute Progressive Maximal Exercise Test (exhaustion test).

The non-diabetic control ALDH2*2 mice and diabetic ALDH2*2 mice treated with vehicle, EMP, Alda- 1 , and EMP + Alda-1 combination were subjected to the exercise exhaustion test as described earlier [10]. We calculated running distance of the mice from the recording.

\section{Post-exercise echocardiography}

Immediately after finishing the exercise exhaustion test, we performed conscious echocardiography again on the mice to record their functional changes. We calculated heart rate, fractional shortening $(\% \mathrm{FS})$ and \% ejection fraction (\%EF) for systolic functional parameters and, relaxation rate (RR) and left atrial (LA) area for diastolic dysfunction.

\section{Histopathology}

The middle portions of the cardiac tissue were fixed with $10 \%$ formalin in PBS, embedded in paraffin as blocks, and several transverse sections were cut for histopathological studies. 


\section{Measurement of cardiomyocyte hypertrophy}

The myocardial sections were stained with hematoxylin-eosin (H-E). H-E-stained cardiac sections were used to measure cardiomyocyte diameter to determine hypertrophy as we reported earlier $[8,9]$. We randomly picked 4 samples from each group to measure cardiomyocyte diameter.

\section{Measurement of cardiac fibrosis}

The myocardial sections were stained with Mason's Trichrome. The red color indicates the deposition of collagen and this area was measured using the MicroSuite software (Olympus America). The percent (\%) area of fibrosis was quantified from each tissue section as previously described $[9,24]$. We randomly picked 4 samples from each group to measure cardiac fibrosis.

\section{ALDH2 activity assay}

The cardiac ALDH2 activity was measured as we described in previous reports $[8,15]$. In brief, the protein samples were allowed to react with $50 \mathrm{mM}$ sodium pyrophosphate as a buffer, $2.5 \mathrm{mM} N A D+$ as a cofactor and $10 \mathrm{mM}$ acetaldehyde as substrate. The reductive reaction of NAD + to NADH at $\lambda 340 \mathrm{~nm}$ wavelength at $37^{\circ} \mathrm{C}$ temperature was measured as ALDH2 activity.

We randomly picked 4 samples from each group to measure ALDH2 activity.

\section{Western immunoblotting}

The Western blot was performed as described earlier $(6,13)$. In brief, protein samples from cardiac tissue were separated on SDS-polyacrylamide gels by electrophoresis and transferred to immobilon-P membranes (Millipore, Billerica, MA). The changes in the protein levels in cardiac tissue samples were determined using antibodies of anti-4HNE-Cys/His/Lys rabbit antibody (Millipore), anti-liver kinase B1 (LKB1) antibody (Cell Signaling Technology), anti-phospho LKB1 (Ser428) antibody (Cell Signaling Technology), AMP-activated protein kinase (AMPK), anti-phospho AMP-activated protein kinase (Thr 172) antibody (Cell Signaling Technology), along with anti-GAPDH antibody (Santacruz) that was used as a housekeeping marker and loading control. The horseradish peroxidase (HRP)-coupled respective secondary antibodies were added to form complexes with primary antibodies which were then visualized by using chemiluminescence detection reagents. We randomly picked 4 samples from each group to do Western immunoblotting.

Immunohistochemistry staining of $80 \mathrm{HdG}$

Formalin-fixed, paraffin-embedded cardiac tissue sections were used for immunohistochemical staining. After deparaffinization and hydration, the slides were washed in Tris-buffered saline (TBS; $10 \mathrm{mmol} / \mathrm{L}$ Tris- $\mathrm{HCl}, 0.85 \% \mathrm{NaCl}, \mathrm{pH} 7.5$ ) containing $0.1 \%$ bovine serum albumin (BSA). Endogenous peroxidase activity was quenched by incubating the slides in $0.6 \% \mathrm{H}_{2} \mathrm{O}_{2}$ /methanol. A pressure cooker method was used to retrieve the antigen. In the following steps, reagents from an immunoperoxidase staining kit (Millipore) were used as directed. A solution from the kit was used to block non-specific reactions. After 
overnight incubation with mouse $80 \mathrm{HdG}$ antibody (Abcam, Cambridge, MA) at a concentration of 1:200 and $4^{\circ} \mathrm{C}$, the slides were washed in TBS. Secondary antibody solution and streptavidin peroxidase solution were added and incubated at room temperature. Immunostaining was visualized with chromogen, diaminobenzidine tetrahydrochloride (DAB). After overnight incubation with mouse $80 \mathrm{HdG}$ antibody (Abcam, Cambridge, MA) at a concentration of 1:200 at $4^{\circ} \mathrm{C}$, the slides were washed in TBS. We counted the brown color spots as $80 \mathrm{HdG}$ positivity in the cardiac sections from various groups using light microscope (Olympus) and plotted them as a graph as we reported earlier [24,32]. We randomly picked 4 samples from each group to perform immunostaining of $80 \mathrm{HdG}$.

\section{Immunofluorescence staining of LKB1 and 4HNE adducts}

Formalin-fixed, paraffin-embedded cardiac tissue sections were used for immunostaining as we explained elsewhere [32]. After deparaffinization and hydration, the slides were washed in Tris-buffered saline (TBS; $10 \mathrm{mmol} / \mathrm{L}$ Tris $-\mathrm{HCl}, 0.85 \% \mathrm{NaCl}, \mathrm{pH} 7.5$ ) containing $0.1 \%$ bovine serum albumin (BSA). A pressure cooker method was used to retrieve the antigen. In the following steps, reagents from an immunoperoxidase staining kit (Millipore) were used as directed. A solution from the kit was used to block non-specific reactions. The following antibodies were used for co-immunostaining at a concentration of $1: 100$ and $4^{\circ} \mathrm{C}$ for overnight incubation: anti-LKB1 mouse monoclonal antibody (Thermofischer Scientific Inc) and anti-4HNE-Cys/His/Lys rabbit polyclonal antibody (Millipore Sigma, 303207). The secondary antibodies conjugated with FITC and rhodamine (Thermofischer Scientific Inc) at a concentration of 1:500 at room temperature for 1 hour. Immunofluorescence positive staining was analyzed using an Olympus microscope and an image analyzer. We randomly picked 4 samples from each group to perform co-immunostaining of LKB1 and 4HNE protein adducts.

\section{Statistical analysis}

Data are presented as mean \pm standard error of the mean (S.E.M). We used One-Way ANOVA for group comparisons and the post-hoc analysis was performed using Student T-test. The differences between before and after exercise stress with individual groups were analyzed by using the paired Student t-test. We used Microsoft Excel for all statistical analysis.

\section{Results}

Effects of EMP, Alda-1 and EMP + Alda-1 on HFD-induced body weight (BW) gain and type-2 diabetes mediated hyperglycemia

HFD feeding increased body weight gain in ALDH2*2 mice compared to chow fed ALDH2*2 mice (Fig. 1A). This increase in body weight gain was not reduced by vehicle, EMP, Alda-1 and EMP + Alda-1 treatments (Fig. 1A). As expected, HFD-induced type-2 diabetes in ALDH2*2 mice increased hyperglycemia compared to chow-fed ALDH2*2 mice (Fig. 1B). Among the treated groups, EMP and a combination of EMP and Alda-1 treatments decreased type-2 diabetes-mediated hyperglycemia compared to vehicle (Fig. 1B). However, Alda-1 treatment did not reduce hyperglycemia (Fig. 1B). 
Effects of EMP, Alda-1 and EMP + Alda-1 on HFD-induced type-2 diabetes mediated glucose intolerance

Similar to hyperglycemia, we found increased glucose intolerance in vehicle-treated type-2 diabetic mice compared to chow-fed control mice (Fig. 1C). EMP alone and as well as in combination with Alda-1 significantly improved the glucose tolerance compared to vehicle and Alda-1 (Fig. 1C).

\section{Effects of EMP, Alda-1 and EMP + Alda-1 on improving cardiac function in conscious echocardiography.}

We presented cardiac functional parameters before (Fig. 2A-2E) and after (Fig. 2F-2J) exercise stress. There was no difference in heart rates (Fig. 2A), the systolic functional parameters i.e., \% \%FS (Fig. 2B) and \%EF (Fig. 2C) before exercise stress in vehicle-treated mice compared to control mice. While there was a significant decrease in RR, diastolic functional parameter (Fig. 2D) and increase in LA area, another measure of poor relaxation and ventricular filling in vehicle-treated mice compared to control mice (Fig. 2E) even before exercise stress. Treatments with EMP, Alda-1 and EMP + Alda-1 attenuated diabetesinduced increases in the LA area (Fig. 2E). Most importantly, EMP + Alda-1 was better in decreasing LA area compared to EMP treatment (Fig. 2E). However, none of the treatments have reduced the LA area to the control levels (Fig. 2E).

After exercise stress, the heart rate was significantly increased in control mice and decreased in vehicletreated mice compared to before exercise stress (Fig. 2F). Treatments with EMP, Alda-1 and EMP + Alda-1 combination increased heart rate after exercise stress (Fig. 2F). There was no difference in \% FS (Fig. 2B and Fig. 2G) and \% EF (Fig. $2 \mathrm{C}$ and Fig. $2 \mathrm{H}$ ) in control mice after exercise stress, however, the \% FS (Fig. 2B and Fig. 2G) and \% EF (Fig. 2C and Fig. 2H) were significantly decreased after exercise stress with vehicle treatment. Diabetes-mediated decreases in \% FS and \% EF were attenuated by EMP, Alda-1 and EMP + Alda-1 combination treatments (Fig. 2B \& 2C and Fig. 2G \& 2H). Most importantly, the EMP + Alda-1 combination treatment improved the $\% \mathrm{FS}$ and $\% \mathrm{EF}$ even better than the individual treatment of EMP (Fig. 2B \& 2C and Fig. 2F \& 2G). Strikingly, the RR was reduced, and the LA area was increased in vehicle treatment compared to controls, which was further improved significantly by treatments with EMP, Alda-1 and EMP + Alda-1 combination (Fig. 2D and 2H). Most importantly, EMP + Alda-1 combination treatment improved the RR and LA area better than EMP alone (Fig. 2D and 2H).

\section{Effects of EMP, Alda-1 and EMP + Alda-1 on exercise tolerance}

We found that vehicle treated ALDH2*2 mice with diabetes associated HFpEF ran for shorter distances until exhaustion compared to non-diabetic control ALDH2*2 mice (Fig. 3). Treatment with EMP or Alda-1 and their combination improved the running distance in mice with diabetes associated HFpEF compared to vehicle treatment (Fig. 3). However, the combination of EMP + Alda-1 improved the running capacity of the mice with diabetes associated HFpEF better than EMP alone (Fig. 3).

\section{Effects of EMP, Alda-1 and EMP + Alda-1 on cardiomyocyte hypertrophy}

We found a significant increase in cardiomyocyte hypertrophy in vehicle treated ALDH2*2 mice with HFpEF after type 2 DM in relation to control mice (Fig. 4A-4B and 4F). EMP or Alda-1 significantly reduces 
cardiomyocyte hypertrophy (Fig. 4A-4D and 4F). EMP + Alda-1 induced decrease in cardiomyocyte hypertrophy was significantly higher than EMP or Alda-1 (4A-4E and 4F). This decrease by EMP + Alda-1 was significantly higher in EMP $(p<0.01)$ than Alda-1 $(p<0.05)$.

\section{Effects of EMP, Alda-1 and EMP + Alda-1 on myocardial fibrosis}

We found a significant increase in myocardial fibrosis in vehicle treated ALDH2*2 mice with diabetes associated HFpEF in relation to control (Fig. 5A-5B and 5F). EMP or Alda-1 significantly reduces myocardial fibrosis (Fig. 5A-5D and 5F). EMP + Alda-1 induced decrease in myocardial fibrosis was significantly higher than EMP or Alda-1 (5A-5E and 5F).

\section{Effects of EMP, Alda-1 and EMP + Alda-1 on 8-0HdG}

We found a significant increase in myocardial 8-OHdG, an index of oxidative DNA damage, in vehicle treated ALDH2*2 mice with diabetes associated HFpEF in relation to control (Fig. 6A-6B and 6G). EMP or Alda-1 significantly reduces myocardial 8-OHdG (Fig. 6A-6D and 6G). EMP + Alda-1 induced decrease in myocardial 8-OHdG was significantly higher than EMP or Alda-1 (6A-6E and $6 \mathrm{G}$ ). The negative control in Fig. $6 \mathrm{~F}$ explains the true immunopositivity in $6 \mathrm{~A}$ to $6 \mathrm{E}$.

\section{Effects of EMP, Alda-1 and EMP + Alda-1 on ALDH2 activity}

We found that the myocardial ALDH2 activity was significantly reduced in vehicle-treated ALDH2*2 mice with diabetes associated HFpEF relative to non-diabetic control mice (Fig. 7). EMP, Alda-1 and EMP+ Alda-1 treatments improved ALDH2 activity significantly (Fig. 7). However, the combination of EMP+ Alda-1 potentiated the activation of cardiac ALDH2 activity significantly compared to EMP or Alda-1 alone (Fig. 7). The increase in ALDH2 activity with EMP + Alda-1 treatment was much higher compared to EMP, than Alda-1 $(p<0.05$ vs $p<0.01)$ However, in the individual treatments, Alda- 1 increased the ALDH2 activity more pronounced than EMP ( $p<0.01$ vs $p<0.001)$ (Fig. 7).

\section{Effects of EMP, Alda-1 and EMP + Alda-1 on 4HNE protein adducts}

Cardiac levels of $4 \mathrm{HNE}$ protein adducts were higher in vehicle treated ALDH2*2 diabetic mice with HFpEF relative to non-diabetic control mice (Fig. 8A, 8B \& 8C). These increased cardiac 4HNE levels were attenuated by EMP or Alda-1 treatment (Fig. 8A, 8B \& 8C). Finally, EMP + Alda-1 combination augmented the decrease in $4 \mathrm{HNE}$ protein adduct levels compared to individual treatments with EMP or Alda-1 (Fig. 8A, 8B \& 8C).

\section{Effects of EMP, Alda-1 and EMP + Alda-1 on LKB1-AMPK signaling}

We found a significant decrease in the LKB1 and phospho LKB1 (Ser428) levels with vehicle treatment, which was increased with EMP or Alda-1 (Figs. 9A, 9B, 9C and 9D). However, this LKB1 increase was further significantly augmented with EMP + Alda-1 treatment (Figs. 9A and 9B). The ratio of phospho LKB1 and LKB1 was significantly reduced in vehicle treatment compared to the controls (Figs. 9A and 
9D). Treatment with EMP, Alda-1 and EMP + Alda-1 increased the ratio of phospho LKB1 and LKB1 (Figs. 9A and 9D). We found a significant decrease in AMPK levels with vehicle treatment compared to control (Figs. 9E and 9F). This decrease was not significantly changed with EMP, Alda-1 and EMP + Alda-1 (Figs. 9E and 9F). The phospho AMPK (Thr 172) levels with vehicle treatment (Figs. 9A and 9G) was significantly reduced compared to controls mice. EMP or Alda- 1 as well as a combination of EMP + Alda-1 increased phospho AMPK levels compared to vehicle treatment (Figs. 9A to 9G). In that, EMP + Alda-1 treatment augmented the increase in the phospho AMPK levels significantly even when compared to EMP alone treatment (Figs. 9A to 9G). The ratio of phospho AMPK/AMPK was significantly reduced in vehicle treatment compared to the controls (Figs. 9A and 9H). The ratio of phospho AMPK/AMPK was significantly increased with EMP, Alda-1 and EMP + Alda-1 treatments (Figs. 9A and 9H). EMP + Alda-1 treatment mediated increase was significantly higher compared to EMP or Alda-1 (Figs. 9A and 9H).

\section{Effects of EMP, Alda-1 and EMP + Alda-1 on 4HNE adduction on LKB1}

We found a significant increase in colocalization of LKB1 and 4HNE in the hearts of vehicle treated ALDH2*2 mice with diabetes associated HFpEF compared to the non-diabetic controls implicating increased 4HNE adduction on LKB1 (Fig. 10A, 10B, 10F, 10G, 10K, 10L as well as 10P and 10Q). EMP or Alda-1 significantly decreased 4HNE adduction on LKB1 compared to vehicle treatment (10C, 10D, 10H, $10 \mathrm{I}, 10 \mathrm{M}, 10 \mathrm{~N}$ as well as $10 \mathrm{P}$ and $10 \mathrm{Q})$. The combination of EMP + Alda-1 treatment decreased $4 \mathrm{HNE}$ adduction on LKB1 compared to vehicle as well as EMP or Alda-1 (10B-10Q). It appears that most of this colocalization occur at the coronary endothelial cells and some in blood cells in the blood vessels (10K100).

\section{Discussion}

Combined treatment of EMP + Alda-1 ameliorated the hallmarks of diabetes associated HFpEF such as cardiac diastolic function and exercise capacity as well as decreased cardiomyocyte hypertrophy and myocardial fibrosis more than the individual treatment with EMP in ALDH2*2 mice. This effect was rendered by decreasing cardiac $4 \mathrm{HNE}$ protein adducts and $80 \mathrm{HdG}$ levels significantly with a notable improvement in the LKB1-AMPK signaling along with attenuating 4HNE adduction on LKB1. In the present study, Alda-1 treatment alone improved the cardiac function and exercise tolerance without decreasing the hyperglycemia but increasing $4 \mathrm{HNE}$ detoxification in ALDH2*2 mutant diabetic mice with HFpEF. Thus, 4HNE may be critical in contributing to the HFpEF pathophysiology. Alda-1 exerted greater improvements in cardiac function, exercise tolerance and increasing LKB1 level, phospho LKB1 (ser 428) and their ratio compared to EMP. From our study, we can point out that Alda-1 increased the metabolism of $4 \mathrm{HNE}$, thus protecting the myocardium from HFpEF. However, the combination treatment of EMP + Alda-1 showed greater benefits.

As we picked diabetes associated HFpEF as a model for our study, such comorbidities specific strategy in HFpEF will be beneficial and it also should fit with the emerging concept of precision medicine. Among the global diabetic population, roughly $40 \%$ of the diabetics are Asians [33], equating to 150 million 
people with a high mortality rate. Several studies have indicated that Asians develop diabetes at a younger age compared to their Caucasian counterparts [33]. East Asians with the ALDH2^2 mutation have a higher incidence of cardiovascular and metabolic diseases [34] such as diabetes [35], diabetesinduced neuropathy and vasculopathy [36] and diabetes-induced diastolic dysfunction [37]. Our ALDH2*2 E487K knock-in mutant mice mimic East Asians with ALDH2*2 E487K mutation [38, 39] and we demonstrated that this ALDH2*2 E487K knock-in mutant mice with HFD-fed type-2 diabetes recapitulated major characteristics of HFpEF [10]. We found increased diastolic dysfunction with preserved systolic function and poor exercise intolerance in these ALDH2*2 mice mimicking HFpEF features. The evidence to substantiate this were decreased relaxation rate [the details of its calculation is provided in our previous report [10]] along with preserved EF (>60\%) and FS (>45\%). In fact, in a recent clinical study, ALDH2*2 mutant variant is associated with an increased risk of HFpEF in patients with hypertension, diabetes and coronary heart disease [17]. Most importantly, diabetes was shown to decrease ALDH2 activity in non-ALDH2*2 mutant animals i.e., WT animals as well [32]. Thus, our research findings will be applicable to all patients with metabolic stress, more so for East Asians with ALDH2*2 mutation. We have recently shown that ALDH2*2 mutant diabetic hearts have reduced coronary endothelial density and Alda-1 can reduce the coronary endothelial cell damage in the myocardium [24]. Since HFpEF originates from systemic oxidative stress, inflammation, and endothelial cell dysfunction at the cardiac tissue levels. We presume Alda-1 prevented $4 \mathrm{HNE}$-mediated inflammation and thereby ameliorated HFpEF in the current model. To specifically understand the role of $4 \mathrm{HNE}$ in the coronary endothelial cell damage, we employed mouse coronary endothelial cells in our recent studies $[14,15]$. In fact, this study also displays that increased 4HNE adduction in LKB1 was higher in coronary endothelial cells (Fig. 10).

Similar to Alda-1, EMP alone improved cardiac function and exercise tolerance in the mice with HFpEF but by decreasing hyperglycemia-mediated 4HNE adducts (Fig. 8A-8C). Notably, EMP is gaining importance in T2DM patients as its benefits extend beyond its glucose-lowering action [40-42]. Specifically, its effect which led to improvements in heart failure patients and cardiovascular death is striking in the landmark randomized clinical trial (RCT) EMPA-REG OUTCOME (Empagliflozin Cardiovascular Outcome Event Trial in Type 2 Diabetes Mellitus Patients) [20]. A meta-analysis of RCTs has also confirmed the benefits of EMP [43]. Gupte et al [40] summarized clinical, preclinical and metaanalysis of SGLT2 inhibitors mediated cardiac benefits. Ongoing RCTs are planned to study the effects of EMP in HFpEF $[44,45]$. For instance, SUGAR-DM-HF (Studies of Empagliflozin and Its Cardiovascular, Renal and Metabolic Effects) RCT will check for the effects of empagliflozin on specific pathways in cardiac and renal effects in patients with type 2 diabetes (or pre-diabetes) and HF. In a study using explanted cardiac papillary muscle from murine (diabetic and non-diabetic) and human HF subjects, EMP directly reduced diastolic dysfunction via improving myofilament function with enhanced myofilament phosphorylation [46].

As roughly 130 million diabetics live in the Western Pacific region, with a significant proportion in East Asia including China, Philippines, Japan and Korea [33], a sub analysis of EMP treatment in the Asian population within EMPA-REG OUTCOME was performed; it revealed that EMP was beneficial to Asians as well [47] which underscores our interest to focus on the ALDH2*2 mutation which increases the 
propensity to develop diabetic cardiac dysfunction [37] as well as HFpEF in East Asians [17]. There are few diabetic animal studies where the beneficial cardiovascular effects of EMP were shown $[40,48,49]$ including our previous study in which EMP was shown to improve cardiac function and exercise tolerance in same animal model by diminishing hyperglycemia-mediated 4HNE adducts [18].

The highlight of this study is the evaluation of the EMP + Alda-1 combination mediated pharmacological effects on diabetes associated HFpEF. The addition of Alda-1 to EMP improved cardiac diastolic dysfunction before exercise stress. After the exercise stress, the combination treatment showed improvements in both diastolic and systolic function besides increasing heart rate. At the cell signaling level, the combination treatment was better in increasing ALDH2 activity, reducing 4HNE protein adducts level, improving the LKB1-AMPK signaling pathway i.e., the increase in the Ser428 pLKB1 and Thr172 pAMPK, and decreasing 4HNE adduction on LKB1. Earlier studies implicated that cardiac-specific haplodeficiency of LKB1 in mice fed with HFD and high-sucrose diet causes deleterious effects at multiple levels [50] including: 1) cardiac functional level - diastolic dysfunction and systolic failure; 2) cellular level - apoptosis, hypertrophy, and fibrosis; 3 ) organelle level - mitochondrial dysfunction as well as 4) signaling level - decrease in AMPK phosphorylation. Pertaining to our line of thinking, 4HNE adduction on essential signaling proteins afflicts cardiac tissue and causes abnormal function [50]. For instance, in spontaneously hypertensive rats, 4HNE adduction on LKB1 in hypertrophic myocardium was shown in reducing AMPK phosphorylation [51]. In that report, 4HNE-mediated inhibition of the LKB1/AMPK signaling was established as critical signaling in the hypertrophic process [51]. Furthermore, at the molecular level, modification at Lys-97 by 4HNE alone can inhibit LKB1 [52]. Even though we did not perform such molecular studies in our current study, our interpretation of the results suggest that decreasing 4HNE protein adduct levels by two different pharmacological approaches protects LKB1 from $4 \mathrm{HNE}$ adduction and thus improving its activity as evident from increased phosphorylation of LKB1 at Ser428 and then its substrate, AMPK at Thr172. Therefore, we propose that efficient removal of cardiac tissue 4HNE level by both augmenting ALDH2 activation mediated 4HNE detoxification along with decreasing hyperglycemia-mediated $4 \mathrm{HNE}$ production will be beneficial in ameliorating diabetes associated HFpEF, which can be tested in other HFpEF model where oxidative stress ensues.

In conclusion, 4HNE-protein adduction mediated cell signaling aberrations are critical in HFpEF. Thus, targeting $4 \mathrm{HNE}$ removal from the organ systems can serve as a key strategy to alleviate HFpEF.

\section{Perspectives}

This study shows that combined treatment with EMP, a SGLT2 inhibitor that lowers hyperglycemia and Alda-1, an ALDH2 activator, improved exercise tolerance and diastolic function better than EMP alone in a mouse model of HFpEF associated with diabetes. This was mediated by reduced cardiac $4 \mathrm{HNE}$ protein adduct levels due to decreased 4HNE production via EMP and augmented 4HNE detoxification by Alda-1.

\section{Declarations}


- Ethics approval and consent to participate

All applicable national, and/or institutional guidelines for the care and use of animals were followed

- Consent for publication

Not applicable. All authors have declared their consent for this publication.

\section{- Availability of data and materials}

All the data are within the manuscript. All materials were obtained from commercial vendors.

\section{- Competing interests}

Not applicable

- Funding

SSP was supported by a grant from the National Heart, Lung, and Blood Institute 1R56HL131891-01A1, 1R01HL139877-01A1 and an internal grant from Henry Ford Health System A10249.

\section{- Authors' contributions}

G.P. conducted the study, collected, analyzed, and organized the data. B.R. contributed to the data collection. S.G. contributed to LKB1-AMPK signaling studies. D.E.L., R.A.T., A.G. and P.O. all contributed to experimental planning and manuscript editing. S.S.P. contributed to experimental design, conductance, data analysis and organizing and then, manuscript writing. All authors reviewed the manuscript.

\section{- Acknowledgements}

The Author(s) declare(s) that there is no conflict of interest.

No disclosures to report

\section{References}

1. Valero-Munoz M, Backman W, Sam F: Murine Models of Heart Failure with Preserved Ejection Fraction: a "Fishing Expedition". JACC Basic Trans/ Sci 2017, 2(6):770-789.

2. Jay $\mathrm{D}$, Hitomi $\mathrm{H}$, Griendling KK: Oxidative stress and diabetic cardiovascular complications. Free radical biology \& medicine 2006, 40(2):183-192.

3. Niemann B, Rohrbach S, Miller MR, Newby DE, Fuster V, Kovacic JC: Oxidative Stress and Cardiovascular Risk: Obesity, Diabetes, Smoking, and Pollution: Part 3 of a 3-Part Series. J Am Coll Cardiol 2017, 70(2):230-251. 
4. Mali VR, Palaniyandi SS: Regulation and therapeutic strategies of 4-hydroxy-2-nonenal metabolism in heart disease. Free radical research 2014, 48(3):251-263.

5. Baynes JW, Thorpe SR: Role of oxidative stress in diabetic complications: a new perspective on an old paradigm. Diabetes 1999, 48(1):1-9.

6. Schalkwijk CG, Stehouwer CDA: Methylglyoxal, a Highly Reactive Dicarbonyl Compound, in Diabetes, Its Vascular Complications, and Other Age-Related Diseases. Physiol Rev 2020, 100(1):407-461.

7. Deiva Dham BR, Amita Gowda, Guodong Pan, Arun Sridhar, Xiangqun Zeng, Rajarajan A. Thandavarayan and Suresh Selvaraj Palaniyandi 4-hydroxy-2-nonenal, a lipid peroxidation product, as a biomarker in diabetes and its complications: challenges and opportunities. Free Radical Research 2020.

8. Mali VR, Ning R, Chen J, Yang XP, Xu J, Palaniyandi SS: Impairment of aldehyde dehydrogenase-2 by 4-hydroxy-2-nonenal adduct formation and cardiomyocyte hypertrophy in mice fed a high-fat diet and injected with low-dose streptozotocin. Exp Biol Med (Maywood) 2014, 239(5):610-618.

9. Mali VR, Pan G, Deshpande M, Thandavarayan RA, Xu J, Yang XP, Palaniyandi SS: Cardiac Mitochondrial Respiratory Dysfunction and Tissue Damage in Chronic Hyperglycemia Correlate with Reduced Aldehyde Dehydrogenase-2 Activity. PLoS One 2016, 11(10):e0163158.

10. Pan G MS, Kar A, Gardinier J, Thandavarayan RA, Palaniyandi SS: Type-2 diabetic aldehyde dehydrogenase 2 mutant mice (ALDH 22) exhibiting heart failure with preserved ejection fraction phenotype can be determined by exercise stress echocardiography. Plos one 2018, E 13 ((4): ):e0195796.

11. Csala M, Kardon T, Legeza B, Lizak B, Mandl J, Margittai E, Puskas F, Szaraz P, Szelenyi P, Banhegyi G: On the role of 4-hydroxynonenal in health and disease. Biochim Biophys Acta 2015, 1852(5):826838.

12. Mali VR, Deshpande M, Pan G, Thandavarayan RA, Palaniyandi SS: Impaired ALDH2 activity decreases the mitochondrial respiration in H9C2 cardiomyocytes. Cell Signal 2016, 28(2):1-6.

13. Pan G, Deshpande M, Thandavarayan RA, Palaniyandi SS: ALDH2 Inhibition Potentiates High Glucose Stress-Induced Injury in Cultured Cardiomyocytes. J Diabetes Res 2016, 2016:1390861.

14. Roy B, Palaniyandi SS: Aldehyde dehydrogenase 2 inhibition potentiates 4-hydroxy-2-nonenal induced decrease in angiogenesis of coronary endothelial cells. Cell Biochem Funct 2020, 38(3):290299.

15. Roy B, Sundar K, Palaniyandi SS: 4-hydroxy-2-nonenal decreases coronary endothelial cell migration: Potentiation by aldehyde dehydrogenase 2 inhibition. Vascul Pharmacol 2020, 131:106762.

16. Deshpande M, Mali VR, Pan G, Xu J, Yang XP, Thandavarayan RA, Palaniyandi SS: Increased 4hydroxy-2-nonenal-induced proteasome dysfunction is correlated with cardiac damage in streptozotocin-injected rats with isoproterenol infusion. Cell Biochem Funct 2016, 34(5):334-342.

17. Xia CL, Chu P, Liu YX, Qu XL, Gao XF, Wang ZM, Dong J, Chen SL, Zhang JX: ALDH2 rs671 polymorphism and the risk of heart failure with preserved ejection fraction (HFpEF) in patients with 
cardiovascular diseases. J Hum Hypertens 2020, 34(1):16-23.

18. Pan G, Deshpande M, Pang H, Palaniyandi SS: Precision medicine approach: Empagliflozin for diabetic cardiomyopathy in mice with aldehyde dehydrogenase (ALDH) 2*2 mutation, a specific genetic mutation in millions of East Asians. Eur J Pharmacol 2018, 839:76-81.

19. Palmer SC, Tendal B, Mustafa RA, Vandvik PO, Li S, Hao Q, Tunnicliffe D, Ruospo M, Natale P, Saglimbene $V$ et al: Sodium-glucose cotransporter protein-2 (SGLT-2) inhibitors and glucagon-like peptide-1 (GLP-1) receptor agonists for type 2 diabetes: systematic review and network metaanalysis of randomised controlled trials. BMJ 2021, 372:m4573.

20. Zinman B, Wanner C, Lachin JM, Fitchett D, Bluhmki E, Hantel S, Mattheus M, Devins T, Johansen OE, Woerle HJ et al: Empagliflozin, Cardiovascular Outcomes, and Mortality in Type 2 Diabetes. $N$ Engl J Med 2015, 373(22):2117-2128.

21. Chen $\mathrm{CH}$, Budas GR, Churchill EN, Disatnik MH, Hurley TD, Mochly-Rosen D: Activation of aldehyde dehydrogenase-2 reduces ischemic damage to the heart. Science 2008, 321(5895):1493-1495.

22. Guo Y, Yu W, Sun D, Wang J, Li C, Zhang R, Babcock SA, Li Y, Liu M, Ma M et al: A novel protective mechanism for mitochondrial aldehyde dehydrogenase (ALDH2) in type i diabetes-induced cardiac dysfunction: role of AMPK-regulated autophagy. Biochim Biophys Acta 2015, 1852(2):319-331.

23. Zhang Y, Babcock SA, Hu N, Maris JR, Wang H, Ren J: Mitochondrial aldehyde dehydrogenase (ALDH2) protects against streptozotocin-induced diabetic cardiomyopathy: role of GSK3beta and mitochondrial function. BMC medicine 2012, 10:40.

24. Pan G, Roy B, Palaniyandi SS: Diabetic Aldehyde Dehydrogenase 2 Mutant (ALDH2*2) Mice Are More Susceptible to Cardiac Ischemic-Reperfusion Injury Due to 4-Hydroxy-2-Nonenal Induced Coronary Endothelial Cell Damage. J Am Heart Assoc 2021, 10(18):e021140.

25. Cheng ST, Chen L, Li SY, Mayoux E, Leung PS: The Effects of Empagliflozin, an SGLT2 Inhibitor, on Pancreatic beta-Cell Mass and Glucose Homeostasis in Type 1 Diabetes. PLoS One 2016, 11(1):e0147391.

26. Han JH, Oh TJ, Lee G, Maeng HJ, Lee DH, Kim KM, Choi SH, Jang HC, Lee HS, Park KS et al: The beneficial effects of empagliflozin, an SGLT2 inhibitor, on atherosclerosis in ApoE (-/-) mice fed a western diet. Diabetologia 2017, 60(2):364-376.

27. Benetti E, Mastrocola R, Vitarelli G, Cutrin JC, Nigro D, Chiazza F, Mayoux E, Collino M, Fantozzi R: Empagliflozin Protects against Diet-Induced NLRP-3 Inflammasome Activation and Lipid Accumulation. J Pharmacol Exp Ther 2016, 359(1):45-53.

28. Gomes KM, Bechara LR, Lima VM, Ribeiro MA, Campos JC, Dourado PM, Kowaltowski AJ, MochlyRosen D, Ferreira JC: Aldehydic load and aldehyde dehydrogenase 2 profile during the progression of post-myocardial infarction cardiomyopathy: benefits of Alda-1. Int J Cardiol 2015, 179:129-138.

29. Gomes KM, Campos JC, Bechara LR, Queliconi B, Lima VM, Disatnik MH, Magno P, Chen CH, Brum PC, Kowaltowski AJ et al: Aldehyde dehydrogenase 2 activation in heart failure restores mitochondrial function and improves ventricular function and remodelling. Cardiovasc Res 2014, 103(4):498-508. 
30. Zhao Y, Wang B, Zhang J, He D, Zhang Q, Pan C, Yuan Q, Shi Y, Tang H, Xu F et al: ALDH2 (Aldehyde Dehydrogenase 2) Protects Against Hypoxia-Induced Pulmonary Hypertension. Arterioscler Thromb Vasc Biol 2019, 39(11):2303-2319.

31. Yang XP, Liu YH, Rhaleb NE, Kurihara N, Kim HE, Carretero OA: Echocardiographic assessment of cardiac function in conscious and anesthetized mice. Am J Physiol 1999, 277(5 Pt 2):H1967-1974.

32. Pan G, Deshpande M, Pang H, Stemmer PM, Carruthers NJ, Shearn CT, Backos DS, Palaniyandi SS: 4Hydroxy-2-nonenal attenuates 8-oxoguanine DNA glycosylase 1 activity. J Cell Biochem 2020.

33. Rhee EJ: Diabetes in Asians. Endocrinol Metab (Seoul) 2015, 30(3):263-269.

34. Chen $\mathrm{CH}$, Ferreira JC, Gross ER, Mochly-Rosen D: Targeting aldehyde dehydrogenase 2: new therapeutic opportunities. Physiol Rev 2014, 94(1):1-34.

35. Murata C, Taniyama M, Kuriyama S, Muramatsu T, Atsumi Y, Matsuoka K, Suzuki Y: Meta-analysis of three diabetes population studies: association of inactive ALDH2 genotype with maternal inheritance of diabetes. Diabetes research and clinical practice 2004, 66 Suppl 1:S145-147.

36. Suzuki Y, Taniyama M, Muramatsu T, Higuchi S, Ohta S, Atsumi Y, Matsuoka K: ALDH2/ADH2 polymorphism associated with vasculopathy and neuropathy in type 2 diabetes. Alcohol Clin Exp Res 2004, 28(8 Suppl Proceedings):111S-116S.

37. Wang C, Fan F, Cao Q, Shen C, Zhu H, Wang P, Zhao X, Sun X, Dong Z, Ma X et al: Mitochondrial aldehyde dehydrogenase 2 deficiency aggravates energy metabolism disturbance and diastolic dysfunction in diabetic mice. J Mol Med (Berl) 2016, 94(11):1229-1240.

38. Zambelli VO, Chen $\mathrm{CH}$, Gross ER: Reactive aldehydes: an initial path to develop precision medicine for pain control. Ann Trans/ Med 2015, 3(17):258.

39. Zambelli VO, Gross ER, Chen CH, Gutierrez VP, Cury Y, Mochly-Rosen D: Aldehyde dehydrogenase-2 regulates nociception in rodent models of acute inflammatory pain. Sci Trans/ Med 2014, 6(251):251ra118.

40. Gupte M, Umbarkar P, Lal H: Mechanistic Insights of Empagliflozin-Mediated Cardiac Benefits: Nearing the Starting Line : Editorial to: "Empagliflozin Improves Left Ventricular Diastolic Dysfunction in a Genetic Model of Type 2 Diabetes" by N. Hammoudi et al. Cardiovasc Drugs Ther 2017, 31(3):229-232.

41. Ndefo UA, Anidiobi NO, Basheer E, Eaton AT: Empagliflozin (Jardiance): A Novel SGLT2 Inhibitor for the Treatment of Type-2 Diabetes. P T 2015, 40(6):364-368.

42. Gallo LA, Ward MS, Fotheringham AK, Zhuang A, Borg DJ, Flemming NB, Harvie BM, Kinneally TL, Yeh SM, McCarthy DA et al: Once daily administration of the SGLT2 inhibitor, empagliflozin, attenuates markers of renal fibrosis without improving albuminuria in diabetic $\mathrm{db} / \mathrm{db}$ mice. Sci Rep 2016, 6:26428.

43. Salsali A, Kim G, Woerle HJ, Broedl UC, Hantel S: Cardiovascular safety of empagliflozin in patients with type 2 diabetes: a meta-analysis of data from randomized placebo-controlled trials. Diabetes Obes Metab 2016, 18(10):1034-1040. 
44. Anker SD, Butler J, Filippatos GS, Jamal W, Salsali A, Schnee J, Kimura K, Zeller C, George J, Brueckmann $\mathrm{M}$ et al: Evaluation of the effects of sodium-glucose co-transporter 2 inhibition with empagliflozin on morbidity and mortality in patients with chronic heart failure and a preserved ejection fraction: rationale for and design of the EMPEROR-Preserved Trial. Eur J Heart Fail 2019, 21(10):1279-1287.

45. Abraham WT, Ponikowski P, Brueckmann M, Zeller C, Macesic H, Peil B, Brun M, Ustyugova A, Jamal W, Salsali A et al: Rationale and design of the EMPERIAL-Preserved and EMPERIAL-Reduced trials of empagliflozin in patients with chronic heart failure. Eur J Heart Fail 2019, 21(7):932-942.

46. Pabel S, Wagner S, Bollenberg H, Bengel P, Kovacs A, Schach C, Tirilomis P, Mustroph J, Renner A, Gummert $\mathrm{J}$ et al: Empagliflozin directly improves diastolic function in human heart failure. Eur $\mathrm{J}$ Heart Fail 2018, 20(12):1690-1700.

47. Kaku K, Lee J, Mattheus M, Kaspers S, George J, Woerle HJ, Investigators E-RO: Empagliflozin and Cardiovascular Outcomes in Asian Patients With Type 2 Diabetes and Established Cardiovascular Disease- Results From EMPA-REG OUTCOME((R)). Circ J 2017, 81(2):227-234.

48. Lin B, Koibuchi N, Hasegawa Y, Sueta D, Toyama K, Uekawa K, Ma M, Nakagawa T, Kusaka H, KimMitsuyama S: Glycemic control with empagliflozin, a novel selective SGLT2 inhibitor, ameliorates cardiovascular injury and cognitive dysfunction in obese and type 2 diabetic mice. Cardiovasc Diabetol 2014, 13:148.

49. Habibi J, Aroor AR, Sowers JR, Jia G, Hayden MR, Garro M, Barron B, Mayoux E, Rector RS, WhaleyConnell A et al: Sodium glucose transporter 2 (SGLT2) inhibition with empagliflozin improves cardiac diastolic function in a female rodent model of diabetes. Cardiovasc Diabetol 2017, 16(1):9.

50. Miller EJ, Calamaras T, Elezaby A, Sverdlov A, Qin F, Luptak I, Wang K, Sun X, Vijay A, Croteau D et al: Partial Liver Kinase B1 (LKB1) Deficiency Promotes Diastolic Dysfunction, De Novo Systolic Dysfunction, Apoptosis, and Mitochondrial Dysfunction With Dietary Metabolic Challenge. J Am Heart Assoc 2015, 5(1).

51. Dolinsky VW, Chan AY, Robillard Frayne I, Light PE, Des Rosiers C, Dyck JR: Resveratrol prevents the prohypertrophic effects of oxidative stress on LKB1. Circulation 2009, 119(12):1643-1652.

52. Calamaras TD, Lee C, Lan F, Ido Y, Siwik DA, Colucci WS: Post-translational modification of serine/threonine kinase LKB1 via Adduction of the Reactive Lipid Species 4-Hydroxy-trans-2-nonenal (HNE) at lysine residue 97 directly inhibits kinase activity. The Journal of biological chemistry 2012, 287(50):42400-42406.

\section{Figures}




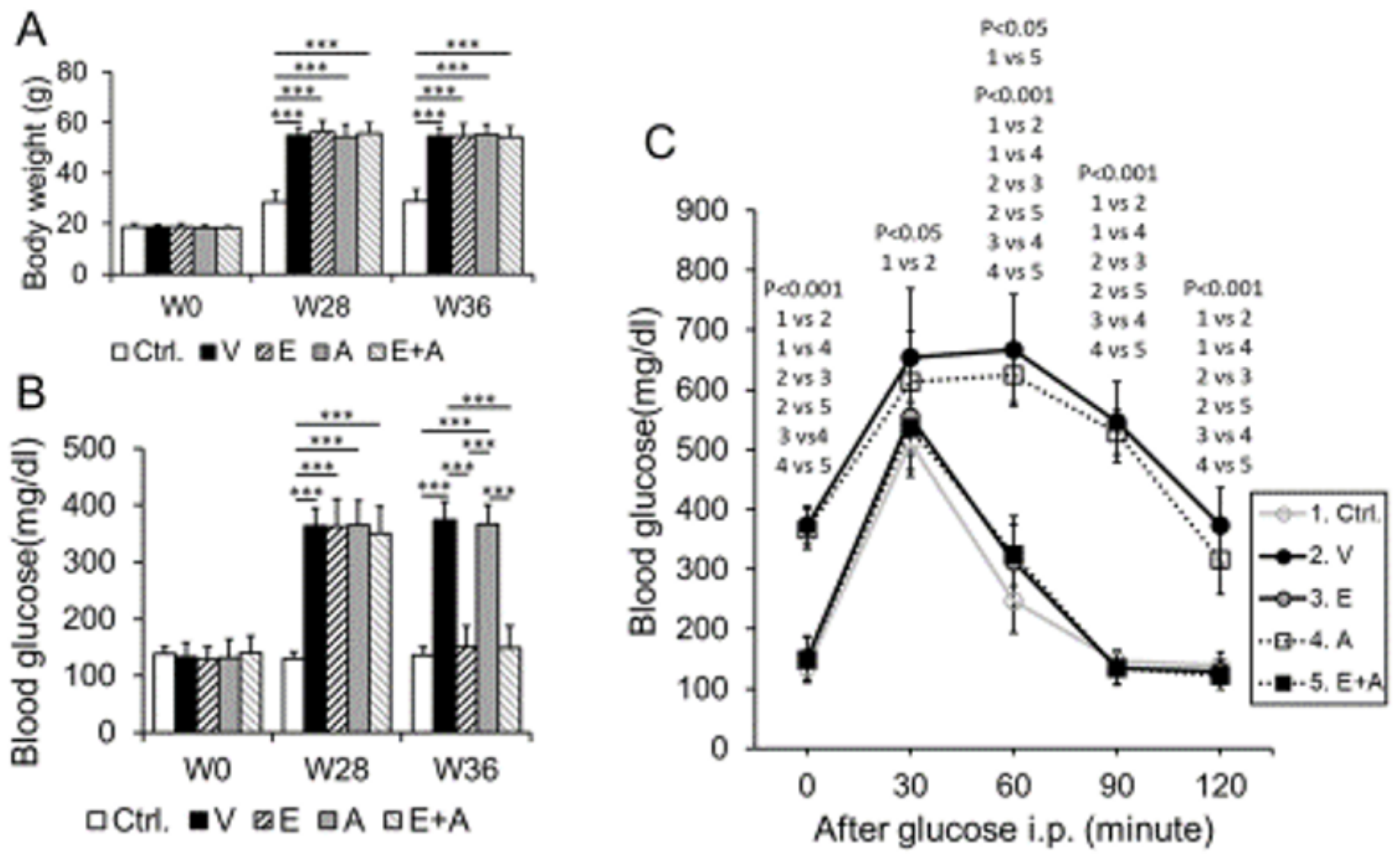

Figure 1

Changes in body weight gain, hyperglycemia and glucose intolerance in diabetic ALDH2*2 mutant mice

Body weight (A), blood glucose (B) and blood glucose tolerance (C) data were shown from non-diabetic control ALDH2 $\star 2$ mutant mice (Ctrl.), vehicle-treated ALDH2 2 mutant diabetic mice $(\mathrm{V}), \mathrm{EMP}$-treated ALDH2*2 mutant diabetic mice (E), Alda-1-treated ALDH2*2 mutant diabetic mice (A) and EMP+ Alda-1treated $A L D H 2 * 2$ mutant diabetic mice $(E+A)$. Data are presented as mean \pm standard error of the mean (SEM). $\mathrm{N}=8$ of each group

In Fig $A,{ }^{* * *} \mathrm{P}<0.001$ vs Ctrl; In Fig $B,{ }^{* \star *} \mathrm{P}<0.001$ vs Ctrl, $\mathrm{E}$ and $\mathrm{A}$ 

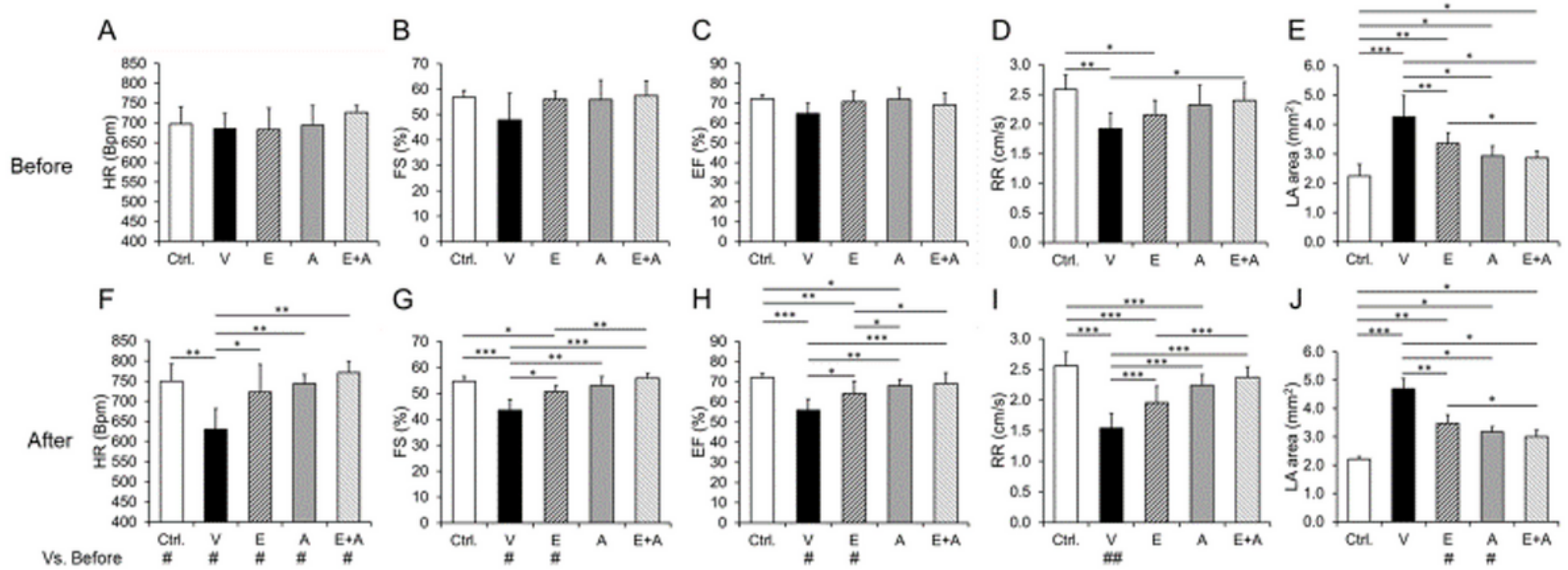

Figure 2

Changes in cardiac functional indices in diabetic ALDH2*2 mutant mice exhibiting HFpEF with vehicle, EMP, Alda-1 and EMP + Alda-1 treatments

Cardiac functional parameters measured before exercise stress: heart rate (HR) (A), \% fractional shortening (FS) (B), \%ejection fraction (EF) (C) and relaxation rate (RR) data (D) and left atrial area (LA area) were shown from non-diabetic control ALDH2*2 mutant mice (Ctrl.), vehicle-treated ALDH2*2 mutant diabetic mice $(\mathrm{V})$, EMP-treated ALDH2*2 mutant diabetic mice (E), Alda-1 treated ALDH2*2 mutant diabetic mice $(A)$ and EMP and Alda-1 combinedly treated ALDH2*2 mutant diabetic mice $(E+A)$. The cardiac functional parameters changes after exercise stress were shown as below: heart rate (HR) $(F)$, \% fractional shortening (FS) (G), \%ejection fraction (EF) (H) and relaxation rate (RR) data (I) and left atrial area (LA area) $(\mathrm{J})$. Data are presented as mean \pm standard error of the mean (SEM). $N=8$ per group.

${ }^{*} \mathrm{P}<0.05,{ }^{* *} \mathrm{P}<0.01$ and ${ }^{* \star *} \mathrm{P}<0.001$ refer to the difference between individual groups as shown in before and after exercise stress.

${ }^{\#} \mathrm{P}<0.05$ and ${ }^{\# \#} \mathrm{P}<0.01$ vs before exercise stress of that particular group. 


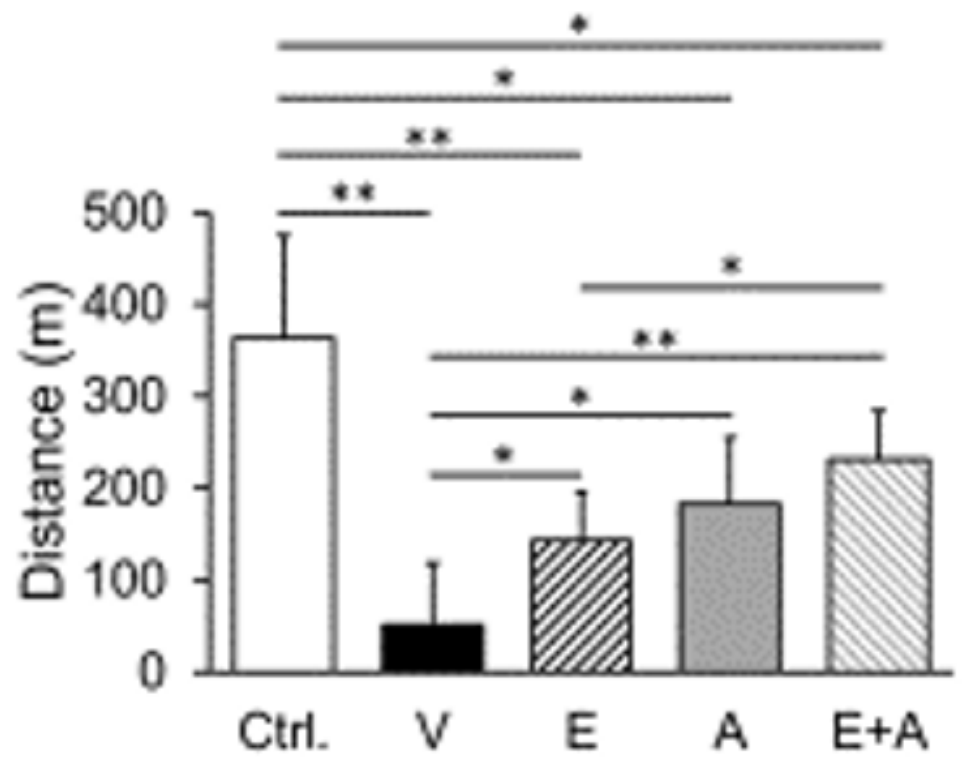

Figure 3

Changes in the running distance with treadmill exercise in diabetic ALDH2*2 mutant mice exhibiting HFpEF with vehicle, EMP, Alda-1 and EMP + Alda-1 treatments

Running distance was shown from non-diabetic control ALDH2*2 mutant mice (Ctrl.), vehicle-treated ALDH2*2 mutant diabetic mice (V), EMP-treated ALDH2*2 mutant diabetic mice (E), Alda-1 treated ALDH2*2 mutant diabetic mice (A) and EMP and Alda- 1 combinedly treated ALDH2*2 mutant diabetic mice $(E+A)$. Data are presented as mean \pm standard error of the mean (SEM). $N=8$

${ }^{*} \mathrm{P}<0.05$ and ${ }^{* \star} \mathrm{P}<0.01$ refer to the difference between individual groups as shown. 

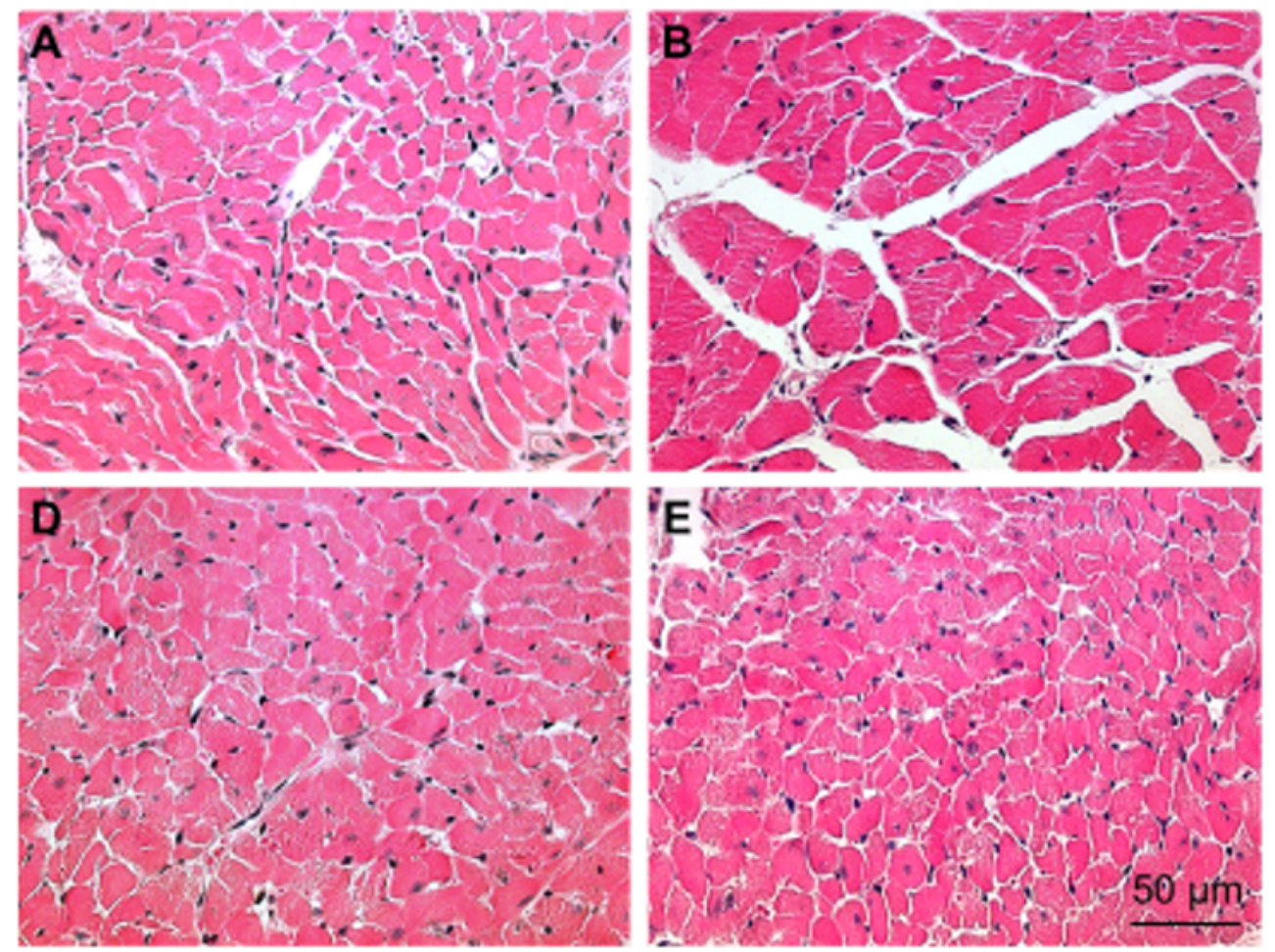
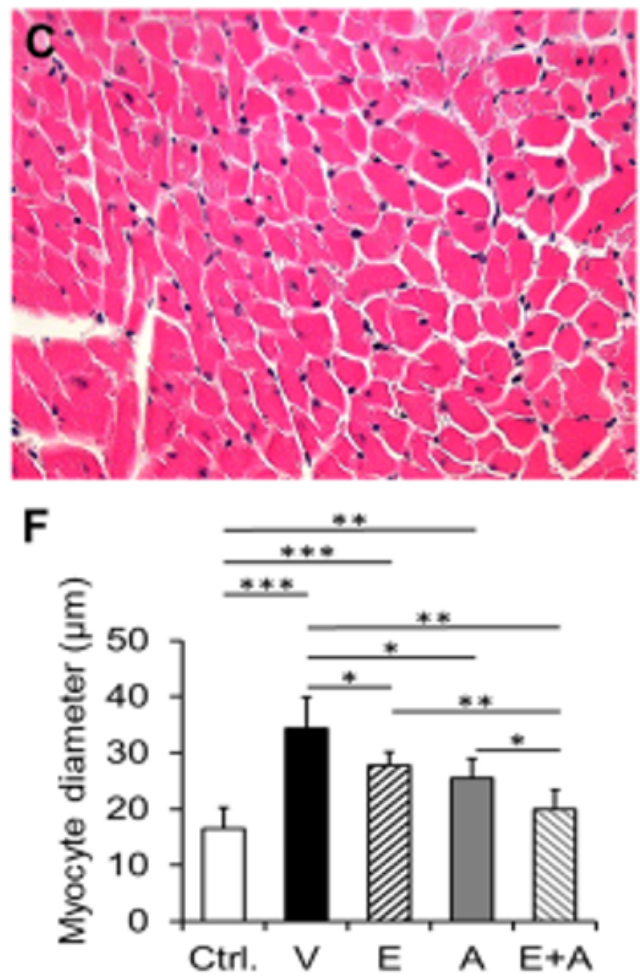

Figure 4

Changes in cardiomyocyte hypertrophy in diabetic ALDH2*2 mutant mice exhibiting HFpEF with vehicle, EMP, Alda-1 and EMP + Alda-1 treatments

Representative micrographs of hematoxylin-eosin (H-E) stained sections from control (A), vehicle-treated ALDH2*2 mutant diabetic mice (B), EMP-treated ALDH2*2 mutant diabetic mice (C), Alda- 1 treated ALDH2*2 mutant diabetic mice (D) and EMP and Alda- 1 combinedly treated ALDH2*2 mutant diabetic mice $(E)$. The quantification of cardiomyocyte hypertrophy was shown $(F)$. Data are presented as mean \pm standard error of the mean (SEM). We have randomly picked $\mathrm{N}=4$ from the 8 mice.

${ }^{\star} \mathrm{P}<0.05,{ }^{\star \star} \mathrm{P}<0.01$ and ${ }^{\star \star *} \mathrm{P}<0.001$ refer to the difference between individual groups as shown. 

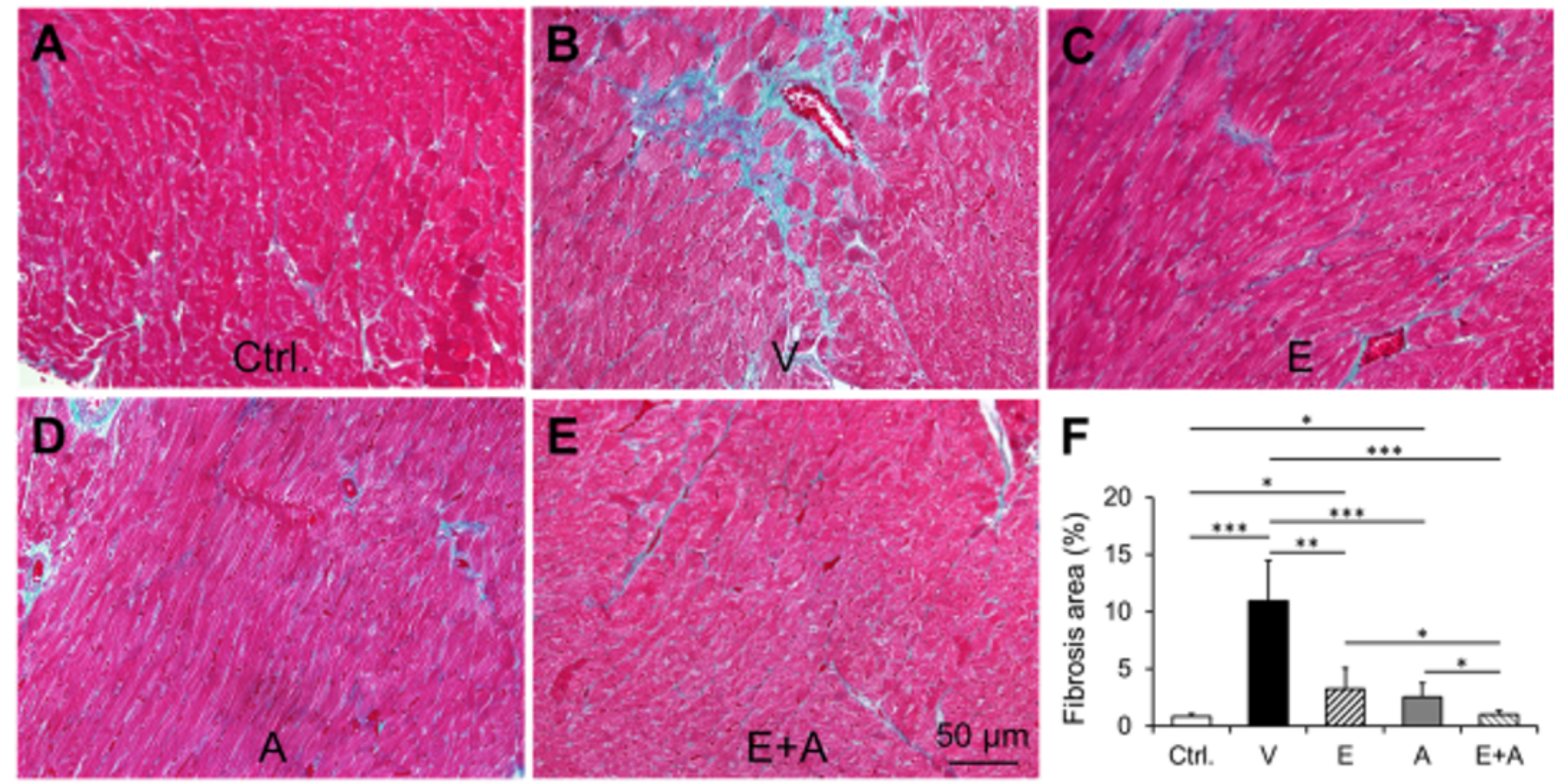

Figure 5

Changes in myocardial fibrosis in diabetic ALDH2*2 mutant mice exhibiting HFpEF with vehicle, EMP, Alda-1 and EMP + Alda-1 treatments

Representative micrographs of Mason Trichrome stained sections from control (A), vehicle-treated ALDH2*2 mutant diabetic mice (B), EMP-treated ALDH2*2 mutant diabetic mice (C), Alda- 1 treated ALDH $2 * 2$ mutant diabetic mice (D) and EMP and Alda- 1 combinedly treated ALDH2*2 mutant diabetic mice $(E)$. The quantification of myocardial fibrosis was shown $(F)$. Data are presented as mean \pm standard error of the mean (SEM). $\mathrm{N}=4$

${ }^{\star} \mathrm{P}<0.05,{ }^{\star \star} \mathrm{P}<0.01$ and ${ }^{\star \star *} \mathrm{P}<0.001$ refer to the difference between individual groups as shown. 

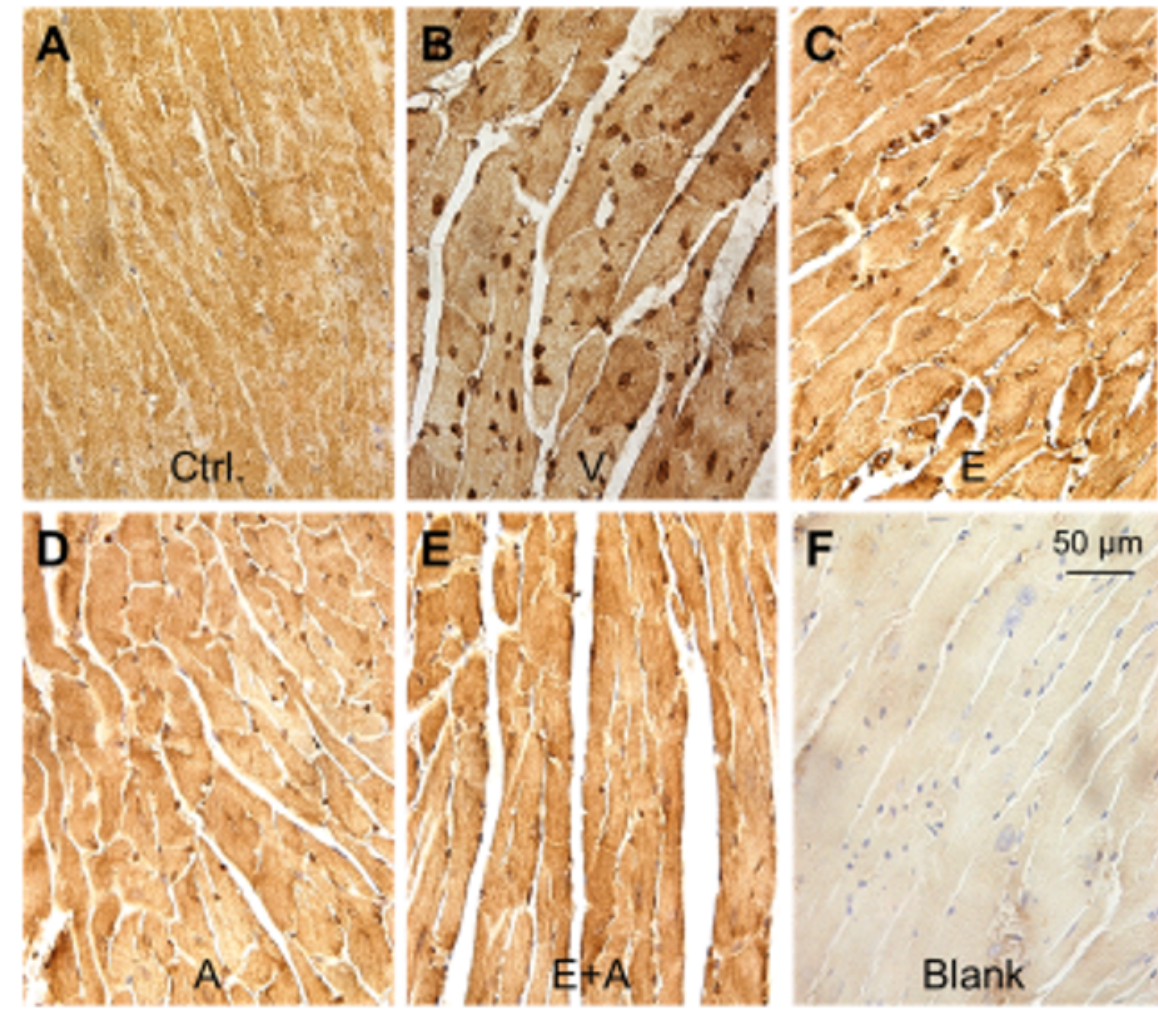

$F$

$50 \mu \mathrm{m}$

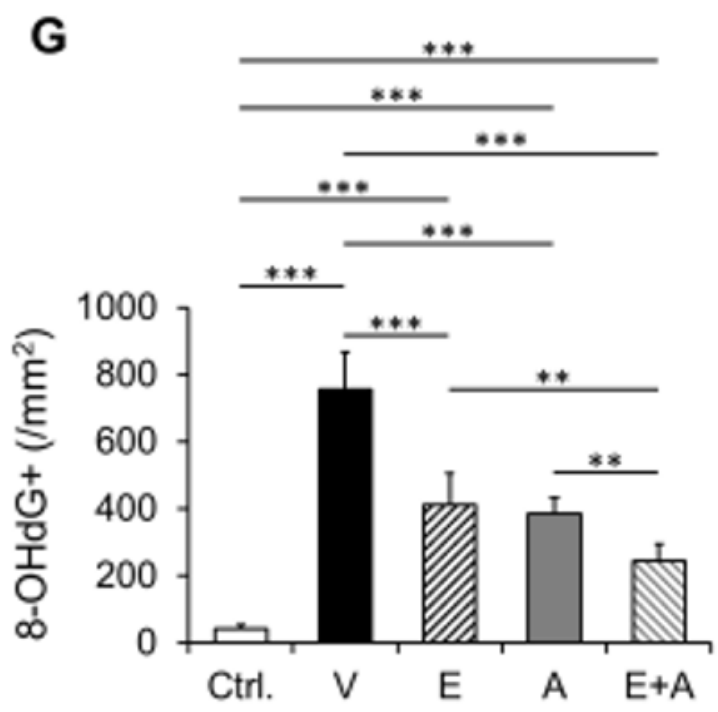

Figure 6

Changes in cardiac $80 \mathrm{HdG}+$ in diabetic ALDH2*2 mutant mice exhibiting HFpEF with vehicle, EMP, Alda-1 and EMP + Alda-1 treatments

Representative micrographs of $80 \mathrm{HdG}$ immunostained sections from control $(\mathrm{A})$, vehicle-treated ALDH2 2 mutant diabetic mice (B), EMP-treated ALDH2*2 mutant diabetic mice (C), Alda- 1 treated ALDH2 $* 2$ mutant diabetic mice (D) and EMP and Alda- 1 combinedly treated ALDH2*2 mutant diabetic mice (E). Blank (negative control) was shown in Fig F. The quantification of $80 \mathrm{HdG}+$ in cardiac tissue was shown (G). Data are presented as mean \pm standard error of the mean (SEM). $N=4$

${ }^{\star \star} \mathrm{P}<0.01$ and ${ }^{\star \star \star} \mathrm{P}<0.001$ refer to the difference between individual groups as shown. 


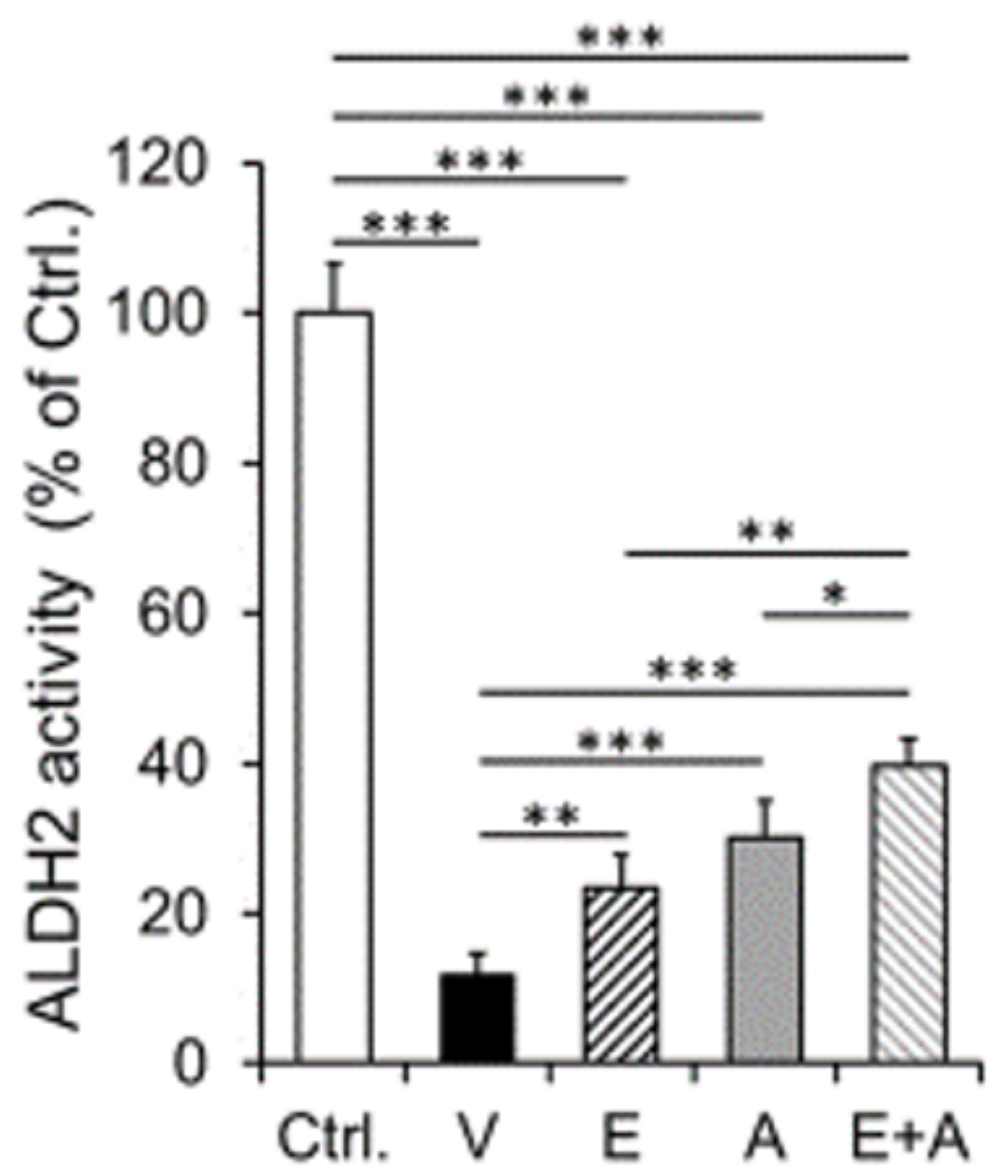

Figure 7

Cardiac ALDH2 activity in diabetic ALDH2*2 mutant mice exhibiting HFpEF with vehicle, EMP, Alda-1 and EMP + Alda-1 treatments

Cardiac ALDH2 activity data of non-diabetic control ALDH2*2 mutant mice (Ctrl.), vehicle-treated ALDH2*2 mutant diabetic mice $(\mathrm{V}), \mathrm{EMP}-\mathrm{treated} \mathrm{ALDH} 2 \star 2$ mutant diabetic mice $(\mathrm{E})$, Alda-1 treated ALDH2*2 mutant diabetic mice (A) and EMP and Alda- 1 combinedly treated ALDH2*2 mutant diabetic mice $(E+A)$ were shown. Data are presented as mean \pm standard error of the mean $(S E M) .{ }^{*} P<0.05$, ${ }^{* \star} \mathrm{P}<0.01$ and ${ }^{* \star *} \mathrm{P}<0.001$ refer to the difference between individual groups as shown. $\mathrm{N}=4$ 
A

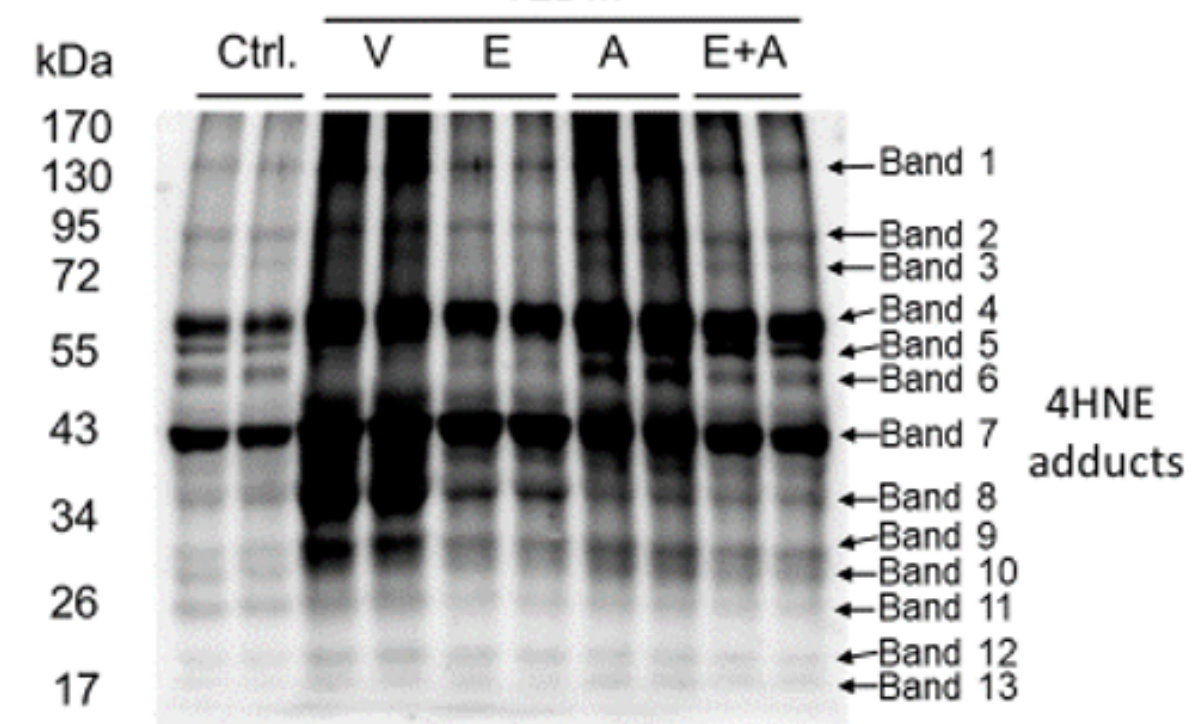

T2DM

GAPDH
B

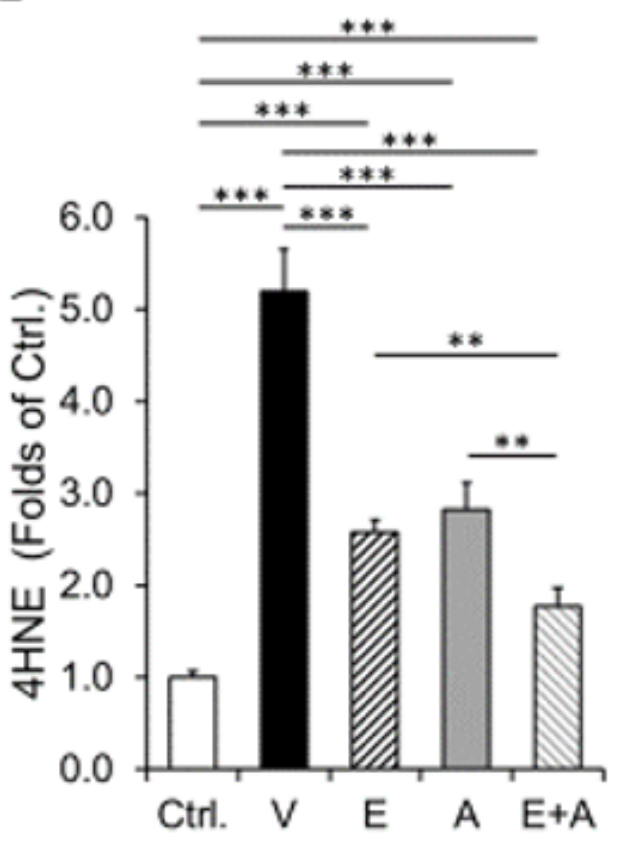

C

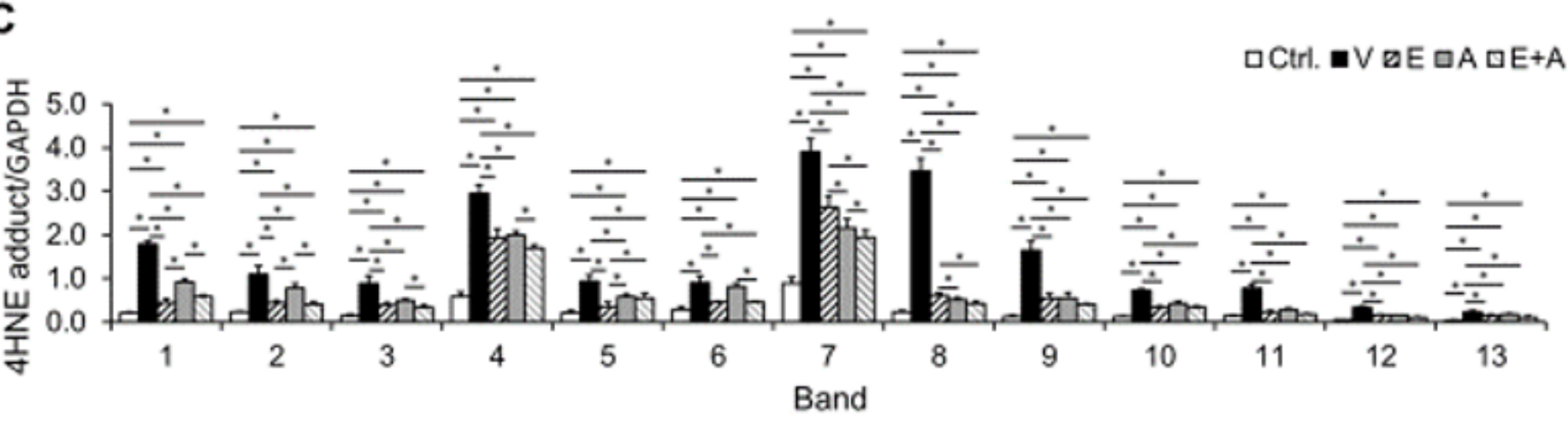

Figure 8

Immunoblotting band images of $4 \mathrm{HNE}$ protein adducts and their quantitation data in diabetic ALDH2*2 mutant mice exhibiting HFpEF with vehicle, EMP, Alda-1 and EMP + Alda-1 treatments

Western blot gel images of $4 \mathrm{HNE}$ protein adducts and the loading control, GAPDH of cardiac tissue samples from Ctrl, $V, E, A$ and $E+A$ groups $(A)$, their densitometric quantification data $(B)$ and the individual $4 \mathrm{HNE}$ protein adduct bands (C) were shown. Data are presented as mean \pm standard error of the mean (SEM). $\mathrm{N}=4$

${ }^{* \star} \mathrm{P}<0.01$ and ${ }^{\star \star *} \mathrm{P}<0.001$ refer to the difference between individual groups as shown in fig $\mathrm{B}$.

${ }^{*} \mathrm{P}<0.05$ refer to the difference between individual groups as shown in fig $\mathrm{C}$. 

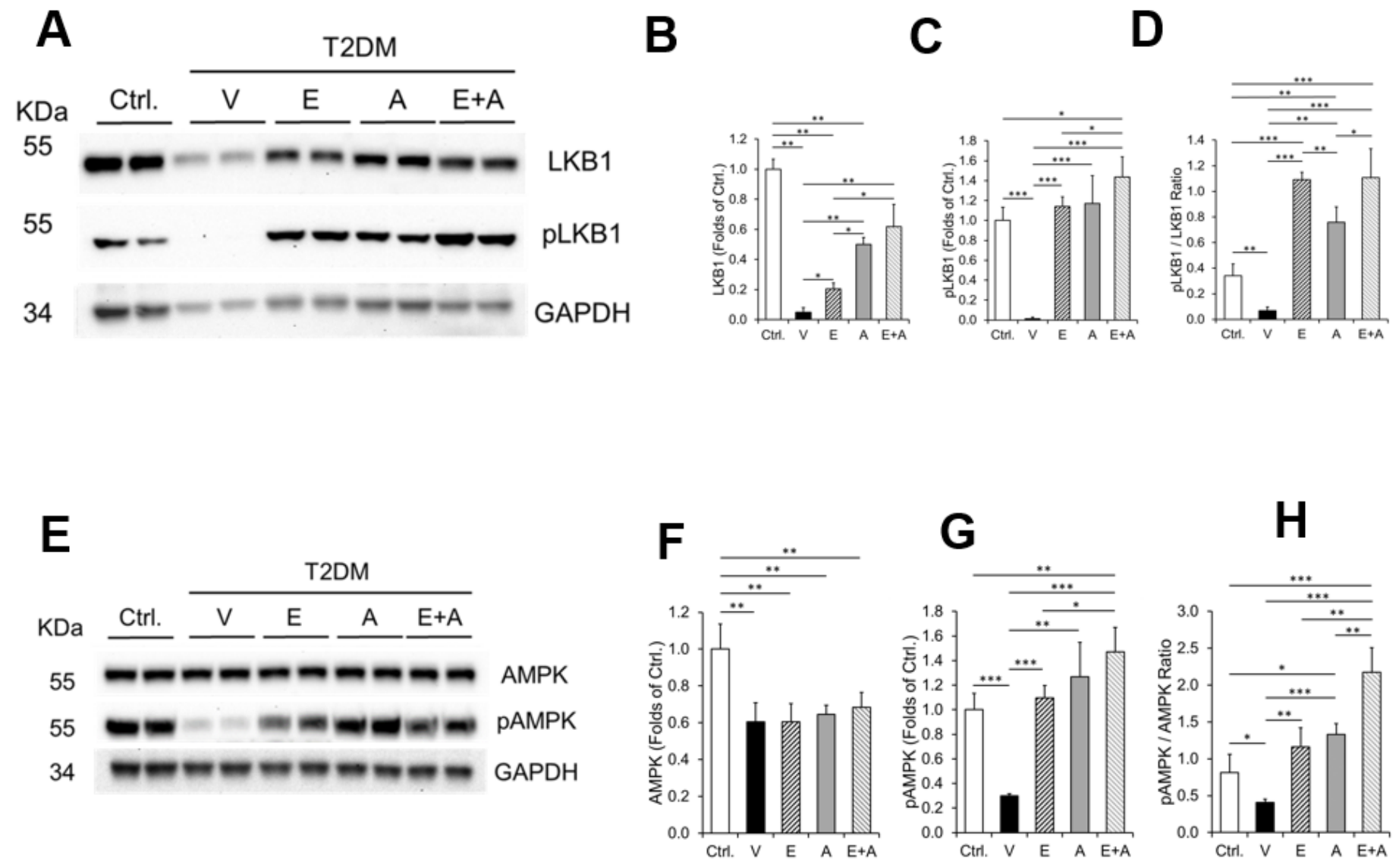

Figure 9

Levels of LKB1, phospho LKB1, phospho LKB1/LKB1, AMPK, phospho AMPK and phospho AMPK/AMPK in diabetic ALDH2*2 mutant mice exhibiting HFpEF with vehicle, EMP, Alda-1 and EMP + Alda-1 treatments

Representative Western blot images of LKB1, phospho LKB1 (pLKB1 i.e., Ser428), and loading control GAPDH (A) and the respective densitometric quantification data of LKB1 (B), pLKB1 (C), pLKB1/LKB1 (D).

Representative Western blot images of AMPK, phospho AMPK (pAMPK i.e., Thr172) and loading control GAPDH (E) and the respective densitometric quantification data of AMPK (F), pAMPK (G) and PAMPK/AMPK $(\mathrm{H})$ were shown.

Data are presented as mean \pm standard error of the mean (SEM). $N=4$

${ }^{* *} \mathrm{P}<0.05,{ }^{* \star} \mathrm{P}<0.01$ and ${ }^{* \star *} \mathrm{P}<0.001$ refer to the difference between individual groups as shown in figures $\mathrm{B}-$ $D$ to $F-G$. 


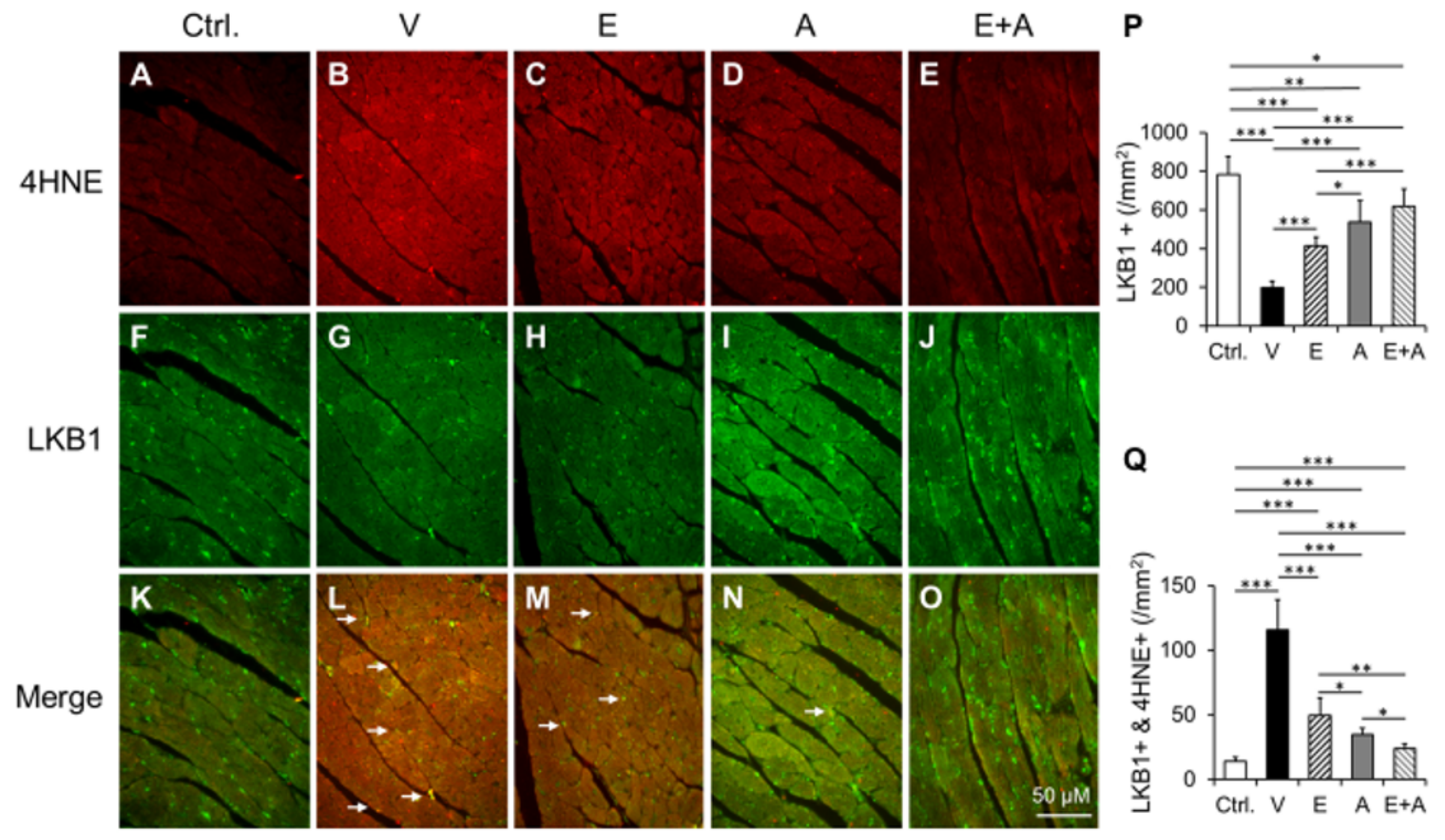

Figure 10

\section{Co-immunostaining of LKB1 and 4HNE protein adducts in the cardiac sections}

Representative micrographs of cardiac sections with immunostaining of LKB1 and 4HNE adducts and their merging from non-diabetic ALDH2*2 control mice (Ctrl) (10A, 10F and 10K), ALDH2*2 diabetic mice exhibiting HFpEF with vehicle (V) (10B, 10G and 10L), (E) (10C, 10H and 10M), (A) (10D, 10I and 10N) and $\mathrm{E}+\mathrm{A}(10 \mathrm{E}, 10 \mathrm{~J}$ and 100$)$ treatments. The white arrows show co-immunopositivity of LKB1 and 4HNE protein adducts. Quantification data of LKB1+ (P) and co-immunostaining of 4HNE with LKB1 (Q) was shown.

Data are presented as mean \pm standard error of the mean (SEM). $N=4$

${ }^{*} \mathrm{P}<0.05,{ }^{* \star} \mathrm{P}<0.01$ and ${ }^{\star \star *} \mathrm{P}<0.001$ refer to the difference between individual groups as shown in figures $\mathrm{P}$ and Q.

\section{Supplementary Files}

This is a list of supplementary files associated with this preprint. Click to download. 
- Supplemental2022221WholemembraneofWBfor5groups.pdf 